
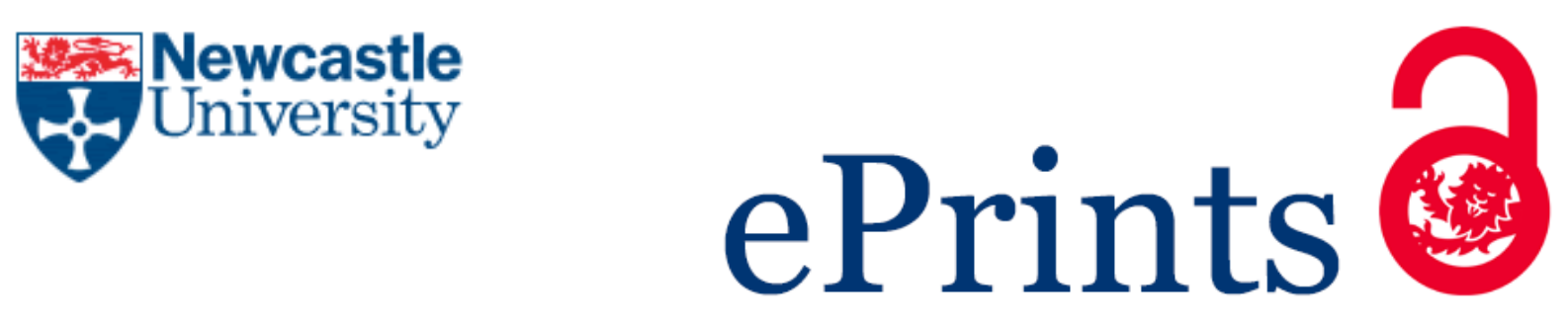

Yigit S, Chen SQ, Quinn P, Chakraborty N.

Numerical investigation of laminar Rayleigh-Bénard convection of Bingham

fluids in square cross-sectioned cylindrical enclosures.

International Journal of Thermal Sciences 2016, 110, 356-368.

\title{
Copyright:
}

C 2016. This manuscript version is made available under the CC-BY-NC-ND 4.0 license

DOI link to article:

http://dx.doi.org/10.1016/i.ijthermalsci.2016.07.013

Date deposited:

$01 / 11 / 2016$

Embargo release date:

04 August 2017

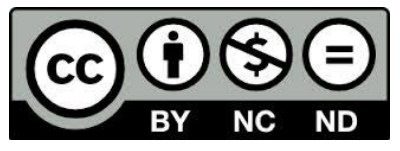

This work is licensed under a

Creative Commons Attribution-NonCommercial-NoDerivatives 4.0 International licence 


\section{Numerical investigation of laminar Rayleigh-Bénard convection of Bingham fluids in square cross-sectioned cylindrical enclosures}

Sahin Yigit ${ }^{1, \bigotimes}$, Siqi Chen ${ }^{1}$, Paul Quinn ${ }^{1}$ and Nilanjan Chakraborty ${ }^{1}$

${ }^{1}$ School of Mechanical and Systems Engineering, Newcastle University, Newcastle-Upon-Tyne, NE1 7RU, UK

${ }^{\circledR}$ Corresponding author.

s.yigit1@ncl.ac.uk

KEYWORDS: Natural convection, non-Newtonian fluid, cylindrical enclosures, yield stress, Bingham model. 


\section{ABSTRACT}

Numerical simulations have been conducted under the assumption of axisymmetry to analyse steady-state laminar natural convection of yield stress fluids obeying Bingham model in square cross-sectioned cylindrical annular enclosures heated from below (i.e. Rayleigh-Bénard configuration). The simulations have been carried out at a representative value of nominal Prandtl number (i.e. $P r=500)$ for different internal cylinder radius $\left(0 \leq r_{i} / L \leq 16\right.$ where $r_{i}$ and $L$ are the inner radius and the cylinder height respectively) for a nominal Rayleigh number range $10^{3} \leq R a \leq 10^{5}$. Both constant wall temperature and constant wall heat flux boundary conditions have been imposed for differentially heated horizontal walls to analyse the effects of wall boundary condition. Although the buoyancy-driven transport strengthens with increasing $R a$, the mean Nusselt number $\overline{N u}_{c y}$ does not show a monotonic increase with increasing $R a$ for small values of $r_{i} / L$ because of the change in flow pattern (i.e. number of convection rolls/cells). By contrast, $\overline{N u}_{c y}$ monotonically increases with increasing $R a$, and only one cell flow pattern is obtained for large values of $r_{i} / L$. Furthermore, $\overline{N u}_{c y}$ has been found to increase with increasing $r_{i} / L$ but asymptotically approaches the corresponding value obtained for square enclosures $\left(r_{i} \rightarrow \infty\right)$ for both CWT and CWHF boundary conditions for large values of $r_{i} / L$. It has also been found that both the flow pattern and the mean Nusselt number $\overline{N u}_{c y}$ are dependent on the initial conditions for Bingham fluid cases since hysteresis is evident for small values of $r_{i} / L$ for both CWT and CWHF boundary conditions. It has been found that convection could be sustained up to a higher value of Bingham number due to stronger convection arising from higher temperature difference between horizontal walls in the case of CWT boundary condition than in the corresponding CWHF configuration. Finally, the numerical findings have been used to propose a correlation for $\overline{N u}_{c y}$ in the range of $2 \leq r_{i} / L \leq 16\left(0.25 \leq r_{i} / L \leq 16\right)$ and $10^{3} \leq$ $R a \leq 10^{5}$ for the CWT (CWHF) boundary condition. 
NOMENCLATURE

\begin{tabular}{|c|c|c|}
\hline$a_{o}$ & {$[-]$} & Correlation parameter \\
\hline$b, b_{o}$ & {$[-]$} & Correlation parameter \\
\hline$B n$ & {$[-]$} & Bingham number \\
\hline$B n_{\max }$ & {$[-]$} & $\begin{array}{l}\text { Bingham number at which or above the mean Nusselt number attains } \\
\text { a value of unity }\end{array}$ \\
\hline$c$ & {$[-]$} & Correlation parameter \\
\hline$c_{p}$ & {$[\mathrm{~J} / \mathrm{kgK}]$} & Specific heat at constant pressure \\
\hline erel & {$[-]$} & Relative error \\
\hline$g$ & {$\left[\mathrm{~m} / \mathrm{s}^{2}\right]$} & Gravitational acceleration \\
\hline$h$ & {$\left[\mathrm{~W} / \mathrm{m}^{2} \mathrm{~K}\right]$} & Heat transfer coefficient \\
\hline$k$ & {$[\mathrm{~W} / \mathrm{mK}]$} & Thermal conductivity \\
\hline$L$ & {$[\mathrm{~m}]$} & height of the enclosure and difference between inner and outer radius \\
\hline$m$ & {$[-]$} & Stress growth exponent \\
\hline$m_{o}$ & {$[-]$} & Correlation parameter \\
\hline$n_{o}$ & {$[-]$} & Correlation parameter \\
\hline$\overline{N u}$ & {$[-]$} & Mean Nusselt number \\
\hline$\overline{N u}_{c y}$ & {$[-]$} & Mean Nusselt number for cylindrical annular enclosure \\
\hline$P$ & {$[\mathrm{~Pa}]$} & Pressure \\
\hline $\operatorname{Pr}$ & {$[-]$} & Prandtl number \\
\hline$q$ & {$\left[\mathrm{~W} / \mathrm{m}^{2}\right]$} & Heat flux \\
\hline$r$ & {$[\mathrm{~m}]$} & Radius \\
\hline$r_{\mathrm{g}}$ & {$[-]$} & Grid expansion ratio \\
\hline$r_{i}$ & {$[\mathrm{~m}]$} & Inner radius \\
\hline$r_{o}$ & {$[\mathrm{~m}]$} & Outer radius \\
\hline$R a$ & {$[-]$} & Rayleigh number \\
\hline$T$ & {$[\mathrm{~K}]$} & Temperature \\
\hline$u$ & {$[\mathrm{~m} / \mathrm{s}]$} & Radial velocity component \\
\hline$u_{i}$ & {$[\mathrm{~m} / \mathrm{s}]$} & $i^{\text {th }}$ velocity component \\
\hline$U$ & {$[-]$} & Dimensionless radial $(U=u L / \alpha)$ velocity component \\
\hline$U_{\text {ref }}$ & {$[\mathrm{m} / \mathrm{s}]$} & Reference velocity scale \\
\hline$w$ & {$[\mathrm{~m} / \mathrm{s}]$} & Vertical velocity component \\
\hline$W$ & {$[-]$} & Dimensionless vertical $(W=w L / \alpha)$ velocity component \\
\hline$z$ & {$[\mathrm{~m}]$} & Coordinate in vertical direction \\
\hline
\end{tabular}




$\begin{array}{lll}\vartheta & {[\mathrm{m} / \mathrm{s}]} & \text { Characteristic velocity } \\ x_{i} & {[\mathrm{~m}]} & \text { Coordinate in } i^{\text {th }} \text { direction } \\ \alpha & {\left[\mathrm{m}^{2} / \mathrm{s}\right]} & \text { Thermal diffusivity } \\ \beta & {[1 / \mathrm{K}]} & \text { Coefficient of thermal expansion } \\ \delta, \delta_{t h} & {[\mathrm{~m}]} & \text { Velocity and thermal boundary-layer thickness } \\ \theta & {[-]} & \text { Dimensionless temperature } \\ \mu & {\left[\mathrm{Ns} / \mathrm{m}^{2}\right]} & \text { Dynamic viscosity } \\ v & {\left[\mathrm{~m}^{2} / \mathrm{s}\right]} & \text { Kinematic viscosity } \\ \rho & {\left[\mathrm{kg} / \mathrm{m}^{3}\right]} & \text { Density } \\ \tau_{\mathrm{ij}}(\tau) & {[\mathrm{Pa}]} & \text { Stress tensor (stress) } \\ \phi & {[-]} & \text { Azimuthal co-ordinate } \\ \Psi & {[-]} & \text { Dimensionless stream function }\end{array}$

\section{Subscripts}

C

cen

cy

eff

$H$

nom

ref

$s q$

\section{Special characters}

$\begin{array}{lll}\Delta T & {[\mathrm{~K}]} & \text { Temperature difference } \\ \Delta_{\text {min,cell }} & {[\mathrm{m}]} & \text { Minimum cell distance }\end{array}$

Cold wall

Geometrical centre

Cylindrical Annular Enclosure

Effective value

Hot wall

Nominal value

Reference value

Square 


\section{INTRODUCTION}

Natural convection of Newtonian fluids in rectangular enclosures has been extensively analysed (see Refs.[1,2] and references therein) but relatively limited attention has been given to natural convection of non-Newtonian fluids despite their wide relevance in many applications such as geophysics, power systems, cooling of electronics. Yield stress fluid is a special type of non-Newtonian fluid, which acts as a solid and does not flow until a threshold yield stress is surpassed. Materials such as mud-slurries in oil drilling, toothpaste, molten chocolate and anti-drip paints are common examples of yield fluids. Aqueous solutions of Carbopol is often used as a model yield stress fluid in laboratory experiments [3-5]. Some magneto- and electro-rheological fluids exhibit yield stress behaviour, and it is possible to modify the yield stress by applying electrical or magnetic fields [6]. This may be useful in reducing heat loses or designing new adaptive thermal systems to control convective thermal transfer in enclosed spaces (i.e. preservations of canned food, chemical processing, storage of cryogenics, solar and nuclear power systems). Several recent semi-analytical [7-10], numerical [3,11-19] experimental studies [3-5] have analysed natural convection of yield stress fluids in rectangular enclosures. These analyses indicated that the buoyancy-driven transport of yield stress fluids weakens with increasing Bingham number (i.e. non-dimensional yield stress) due to additional flow resistance arising from yield stress. This leads to a reduction in convective heat transfer rate, and thermal transport takes place purely due to conduction for large values of Bingham numbers since fluid flow practically stops under such a condition. A qualitatively similar behaviour can be expected for cylindrical annular enclosures but the heat transfer rate for a given set of nominal values of Rayleigh, Prandtl and Bingham numbers might be quantitatively different in comparison to that for rectangular enclosures since curvature of the curved walls in cylindrical enclosures can potentially influence the heat and momentum transport. Although cylindrical annular space is more relevant to real engineering applications than rectangular enclosures (i.e. 
cylindrical cryogenic storages), laminar natural convection of yield stress fluids in cylindrical annular enclosures has not been analysed in detail in existing literature. This gap in existing literature has been addressed here by numerically analysing natural convection of Bingham fluids in square cross-sectioned cylindrical annular enclosures heated from below for the range of nominal Rayleigh number $10^{3} \leq R a \leq 10^{5}$ at a representative value of nominal Prandtl number (i.e. $\operatorname{Pr}=\mu c_{p} / k=500$ ) for $0 \leq r_{i} / L \leq 16$ where $r_{i}$ and $L$ are the internal radius and the cylinder height respectively. The main objectives of this study are:

i) To demonstrate the effects of $R a$ and $B n$ on laminar Rayleigh-Bénard convection of Bingham fluids in square cross-sectioned cylindrical annular enclosures for different values of $r_{i} / L$.

ii) To provide physical explanations for the observed $R a, B n$ and $r_{i} / L$ dependence of mean Nusselt number in the aforementioned configuration.

The rest of the paper will be organised as follows. The mathematical background behind this work will be discussed in the next section. This will be followed by the presentation of results and the subsequent discussion. The main findings will be summarised and conclusions will be drawn in the final section of this paper.

\section{MATHEMATICAL BACKGROUND}

The Bingham model is the simplest model which describes the interrelation between the shear sress and strain rate in yield stress fluids [20]. The strain rate dependence of viscous stresses for Bingham fluids (i.e. fluids obeying the Bingham model) can be expressed as:

$$
\begin{array}{lll}
\underline{\dot{\gamma}}=0 & \text { for } & \tau \leq \tau_{y}, \\
\underline{\underline{\tau}}=\left(\mu+\tau_{y} / \dot{\gamma}\right) \underline{\underline{\underline{\gamma}}} & \text { for } & \tau>\tau_{y},
\end{array}
$$

where $\dot{\gamma}_{i j}=\left(\partial u_{i} / \partial x_{j}+\partial u_{j} / \partial x_{i}\right)$ are the components of the rate of strain tensor $\underline{\underline{\gamma}}, \underline{\underline{\tau}}$ is the stress tensor, $\tau_{y}$ is the yield stress, $\mu$ is the plastic viscosity, $\tau$ and $\dot{\gamma}$ are the second invariants 
of the stress and the rate of strain tensor in a pure shear flow respectively, which are expressed as:

$$
\begin{aligned}
& \tau=\left[\frac{1}{2} \underline{\underline{\tau}}: \underline{\underline{\tau}}\right]^{1 / 2}, \\
& \dot{\gamma}=\left[\frac{1}{2} \underline{\underline{\underline{\gamma}}}: \underline{\underline{\gamma}}\right]^{1 / 2} .
\end{aligned}
$$

For the current analysis the bi-viscosity regularisation proposed by O'Donovan and Tanner [21] has been used to mimic the shear rate dependence of viscous stress for a Bingham fluid:

$$
\begin{array}{ll}
\underline{\underline{\tau}}=\mu_{\text {yield }} \underline{\underline{\gamma}} & \text { for } \quad \dot{\gamma} \leq \tau_{y} / \mu_{\text {yield }}, \\
\underline{\underline{\tau}}=\tau_{y}(\underline{\underline{\gamma}} / \dot{\gamma})+\mu \underline{\underline{\underline{\gamma}}} & \text { for } \quad \dot{\gamma}>\tau_{y} / \mu_{\text {yield }},
\end{array}
$$

where $\tau_{y}$ is the yield stress tensor, $\mu_{y i e l d}$ is the yield viscosity, and , $\mu$ is the plastic viscosity. O'Donovan and Tanner [21] indicated that a value of $\mu_{y i e l d}$ equal to $1000 \mu$ mimics the true Bingham model in a satisfactory manner but here $\mu_{y i e l d} / \mu=10^{4}$ is taken to ensure high fidelity of the simulations. Here, a limited number of simulations have also been carried out based on the regularization proposed by Papanastasiou [22] in order to assess the sensitivity of the simulations on the choice of regularization. Papanastasiou's regularisation [22] takes the following form:

$$
\underline{\underline{\tau}}=\underline{\underline{\tau_{y}}}(1-\exp (-m \dot{\gamma}))+\mu \underline{\underline{\gamma}}
$$

where $m$ is the stress growth exponent which has the dimension of time (i.e. $L^{2} / \alpha$ ). The value of $m$ is taken as $m \gg 10^{5} L^{2} / \alpha$ to ensure the true Bingham model in a satisfactory manner. Both Eqs. 5 and 6 transform the "unyielded" region to a zone of high viscosity. The typical difference between the mean Nusselt number obtained from these two regularizations remains of the same order as typical experimental/numerical uncertainties $(\sim 2 \%)$. 
The schematic diagram of the configuration is shown in Fig.1, which demonstrates that both constant wall temperature (CWT) constant wall heat flux (CWHF) boundary conditions have been considered for the differentially heated horizontal walls while the vertical walls are assumed to be adiabatic. According to Buckingham's pi theorem, it is possible to show that the Nusselt number for natural convection of Bingham fluids in square cross-sectioned cylindrical annular enclosures can be expressed as: $N u=f_{1}\left(\operatorname{Ra}, \operatorname{Pr}, B n, r_{i} / L\right)$, where the nominal Rayleigh, Prandtl and Bingham numbers for Bingham fluids can be defined for CWT and CWHF boundary configurations in the following manner:

\section{$\underline{\text { For CWT }}$}

$R a=\rho g \beta\left(T_{H}-T_{C}\right) L^{3} / \mu \alpha$ and $B n=\tau_{y} L / \mu \sqrt{g \beta\left(T_{H}-T_{C}\right) L}$

\section{For CWHF}

$R a=\rho g \beta q L^{4} / k \mu \alpha$ and $B n=\tau_{y} / \mu \sqrt{g \beta q / k}$

The Bingham number represents the ratio of yield stress to viscous stress. The current analyses have been carried out for a single representative value of nominal Prandtl number (i.e. $\operatorname{Pr}=$ $\left.\mu c_{p} / k=500\right)$ since practical yield stress fluids exhibit Prandtl numbers order of 100 [3-5]. For example, a recent experimental analysis [5] based on experimental analysis of RayleighBénard convection of yield stress fluids in rectangular enclosures reported that $0.05 \%$ by mass Carpool solution in water shows a yield stress behaviour with a nominal Prandtl number of $\operatorname{Pr} \approx 350$.

The local heat transfer coefficient $h$ is defined as:

$h=\left|-k\left(\frac{\partial T}{\partial z}\right)_{z=0} \times 1 /\left(T_{z=0}-T_{z=L}\right)\right|$

The mean heat transfer coefficient $\bar{h}$ and the mean Nusselt number $\overline{N u}_{c y}$ are evaluated as:

$\bar{h}=\int_{r_{i}}^{r_{i}+L} 2 \pi h(r) d r /\left[\pi\left(r_{i}+L\right)^{2}-\pi r_{i}^{2}\right], \quad \overline{N u}_{c y}=\bar{h} L / k$ 
The current analysis has been carried out in non-dimensional form for the sake of generalisation. The spatial co-ordinates, velocity components, pressure and temperature can be non-dimensionalised in the following manner:

$r_{i}^{+}=r_{i} / L, z^{+}=z / L, u_{i}^{+}=u_{i} / U_{\text {ref }}, P^{+}=P / \rho U_{\text {ref }}^{2}, \Theta=\left(T-T_{\text {ref }}\right) / \Delta T_{\text {ref }}$

where the reference velocity scale $U_{\text {ref }}$ is taken to be equal to $\sqrt{g \beta \Delta T_{\text {ref }} L}$ based on the equilibrium of inertial and the buoyancy forces [11-18] where $\Delta T_{r e f}$ is a reference temperature difference. For CWT configuration $\Delta T_{r e f}$ can be taken to be $\Delta T=T_{H}-T_{C}$ while it can be taken to be $\Delta T_{r e f}=q L / k$ for the CWHF configuration. Additionally, the reference temperature is taken to be temperature at the centre of the domain $T_{c e n}$ for the CWHF boundary condition, whereas it is taken to be the cold wall temperature $T_{C}$ for the CWT boundary condition. Accordingly, the steady-state non-dimensional forms of governing equations for mass, momentum and energy for constant temperature-independent thermo-physical properties take the following form under the assumption of axisymmetry:

Non-dimensional mass conservation equation:

$\frac{1}{r^{+}} \frac{\partial\left(r^{+} u^{+}\right)}{\partial r^{+}}+\frac{\partial w^{+}}{\partial z^{+}}=0$

\section{Non-dimensional momentum conservation equations}

Radial direction:

$u^{+} \frac{\partial u^{+}}{\partial r^{+}}+w^{+} \frac{\partial u^{+}}{\partial z^{+}}=-\frac{\partial P^{+}}{\partial r^{+}}+\frac{P r^{1 / 2}}{R a^{1 / 2}}\left[\frac{1}{r^{+}} \frac{\partial\left(r^{+} \tau_{r r^{+}}\right)}{\partial r^{+}}-\frac{\tau_{\phi \phi^{+}}}{r^{+}}+\frac{\partial\left(\tau_{r z}{ }^{+}\right)}{\partial z^{+}}\right]$

Vertical direction:

$u^{+} \frac{\partial w^{+}}{\partial r^{+}}+w^{+} \frac{\partial w^{+}}{\partial z^{+}}=-\frac{\partial P^{+}}{\partial z^{+}}+\Theta+\frac{P r^{1 / 2}}{R a^{1 / 2}}\left[\frac{1}{r^{+}} \frac{\partial\left(r^{+} \tau_{r z}{ }^{+}\right)}{\partial r^{+}}+\frac{\partial\left(\tau_{z z}{ }^{+}\right)}{\partial z^{+}}\right]$

Non-dimensional energy conservation equation:

$u^{+} \frac{\partial \Theta}{\partial r^{+}}+w^{+} \frac{\partial \Theta}{\partial z^{+}}=\frac{1}{P r^{1 / 2} R a^{1 / 2}}\left[\frac{1}{r^{+}} \frac{\partial}{\partial r^{+}}\left(r^{+} \frac{\partial \Theta}{\partial r^{+}}\right)+\frac{\partial^{2} \Theta}{\partial z^{+} \partial z^{+}}\right]$ 
In Eq. $12 \tau_{i j}{ }^{+}$is the non-dimensional stress tensor which is given by:

$\tau_{i j}^{+}=\tau_{i j} L / \mu \sqrt{g \beta \Delta T_{r e f} L}$

where $r$ is the radial coordinate, $z$ axis is taken to align with the vertical direction, and the axisymmetric flow is independent of the azimuthal direction $\phi$. The radial and vertical velocity components are given by $u$ and $w$ respectively. However, the strain rate in the circumferential direction $\dot{\gamma}_{\phi \phi}$ takes the form $\dot{\gamma}_{\phi \phi}=u / r$ in axisymmetric configuration [23] and thus $\tau_{\phi \phi}$ appears in Eq. 12i. The components of viscous stress tensor (i.e. $\tau_{r r}, \tau_{\phi \phi}, \tau_{r z}$ and $\tau_{z z}$ ) are expressed according to Eq. 5. Equations 11-14 are solved in a coupled manner in conjunction with the following boundary conditions. The two vertical walls are kept under adiabatic conditions (i.e. $\partial \Theta / \partial r^{+}=0$ at $r^{+}=r_{i} / L$ and $r^{+}=r_{i} / L+1$ ), and both velocity components (i.e. $u^{+}$and $w^{+}$) are identically zero on each boundary because of the no-slip condition and impenetrability of rigid walls. For the CWHF configuration, the heat fluxes for horizontal hot and cold walls are specified (i.e. $-\partial \Theta / \partial z^{+}=1$ at $z^{+}=0.0$ and $z^{+}=1.0$ respectively). By contrast, the temperatures of horizontal walls are specified (i.e. $\Theta=1$ and $\Theta=0$ at $z^{+}=0.0$ and $z^{+}=1.0$ respectively) for CWT configuration.

\section{NUMERICAL IMPLEMENTATION}

The governing equations of mass, momentum and energy have been numerically solved in the context of finite-volume methodology using a commercial package ANSYS-FLUENT. This commercial package was previously used successfully for simulating Bingham fluid flows [1118]. A second-order central difference scheme is used for the discretization of the diffusive terms and a second-order up-wind scheme is used for the convective terms. The well-known SIMPLE [24] (Semi-Implicit Method for Pressure-Linked Equations) algorithm is used for coupling of the pressure and velocity components. The convergence criteria were set to $10^{-6}$ for all the relative (scaled) residuals. Furthermore, grid-independence of $\overline{N u}_{c y}$ and streamline 
pattern for both Newtonian and Bingham fluids has been established based on three different non-uniform meshes M1 $(100 \times 100)$, M2 $(160 \times 160)$, and M3 $(220 \times 220)$. The nondimensional minimum grid spacing $\left(\Delta_{\text {min,cell }} / L\right)$ and grid expansion ratio $\left(r_{g}\right)$ values for the Cartesian meshes are $4.01 \times 10^{-3}, 2.51 \times 10^{-3}, 1.83 \times 10^{-3}$ and $1.016,1.01,1.007$ respectively. It has been ensured that a change in the computational grid did not lead to a change in flow pattern within the enclosure during the course of grid independency analysis. The numerical uncertainty levels for the mean Nusselt number $\overline{N u}_{c y}$ and maximum non-dimensional vertical velocity component $W_{\max }$ on the horizontal mid-plane for meshes M2 and M3 have been found to be smaller than $1 \%$ for the range of $10^{3} \leq R a \leq 10^{5}$ for $r_{i} / L=0-16$ for both Newtonian and Bingham fluids. Therefore, the simulations have been conducted using the mesh M2 in this analysis for the sake of high accuracy and computational efficiency.

The mean Nusselt numbers $\overline{N u}$ for laminar Rayleigh- Bénard convection of Newtonian fluids in square enclosures for $10^{3} \leq R a \leq 10^{6}$ and $\operatorname{Pr}=0.71$ have been compared to the benchmark data [25] in Table 1. It is evident from Table 1 that an excellent agreement has been achieved between the present results and the benchmark data [25]. It is worth noting that the mean Nusselt number reported in Table 1 have been calculated using the heat flux obtained for a second order spatial discretisation scheme and the same approach has been adopted for the rest of this analysis. The maximum difference in the mean Nusselt number values due to first and second order spatial discretisation has been found to be less than $1 \%$ for both Newtonian (i.e. $B n=0$ ) and Bingham fluids. The Bingham fluid simulations also have been compared to the benchmark data reported by Vola et al. [26] for natural convection of Bingham fluids in square enclosures with vertical walls with different uniform temperatures. It is worth noting that Vola et al. [26] reported only the values of yield stress $\tau_{y}$. According to eq. 7 , the yield stress values reported in Table 5 of Vola et al. [26] give rise to $B n=3.0,0.95$ and 0.3 for 
$R a=10^{4}, 10^{5}$ and $10^{6}$ respectively. The mean Nusselt number values obtained for the current numerical methodology for the aforementioned values of $B n$ and $R a$ for $P r=1.0$ (as used in Ref. [26]) are found to be in good agreement with the corresponding values reported by Vola et al. [26] (maximum difference in $\overline{N u}$ is found to be less than 3\%). Furthermore, the same numerical methodology was successfully used in several previous analyses [11-18].

It has been reported in [7] that Bingham fluid flows are unconditionally linearly stable under quiescent starting conditions. Thus, the steady-state solutions for Newtonian (i.e. $B n=0$ ) fluids for a given set of values of nominal Rayleigh and Prandtl numbers are used as the starting conditions for Bingham fluid simulations. Some magneto- and electro-rheological fluids exhibit yield stress behaviour, and it is possible to modify the yield stress by applying electrical or magnetic fields. Therefore, the steady-state Newtonian solutions can be considered as realistic starting conditions with practical relevance for the Bingham fluid simulations.

\section{RESULTS AND DISCUSSION}

\section{Effects of Bingham number}

The contours of non-dimensional temperature $\theta=\left(T-T_{\text {cen }}\right) / \Delta T_{\text {ref }}$ and stream function $\Psi=$ $\psi / \alpha^{1}$ for different values of $B n$ for $r_{i} / L=1, R a=10^{5}$ and $\operatorname{Pr}=500$ in the CWHF configuration are shown in Fig. 2. It is clear from Fig. 2 that the magnitude of $\Psi$ decreases and isotherms become increasingly parallel to the horizontal walls with increasing $B n$. This indicates that convection weakens with increasing $B n$ and thermal transport takes place purely due to conduction for large values of $B n$. The grey regions on the stream function distribution

${ }^{1}$ It is also possible to define the non-dimensional stream function as $\Psi^{\prime}=\psi /(\mu / \rho)$ but this definition remains directly proportional to $\Psi$ (i.e. $\Psi^{\prime}=\psi / v=\Psi / P r$ ) and does not change the physics of problem. The current definition for $\Psi$ (i.e. $\Psi=\psi / \alpha$ ) was used in several previous analyses [11-18]. 
in Fig. 2 indicate the Apparently Unyielded Regions (AURs) (regions where $|\tau| \leq \tau_{y}$ [27]). The AURs can be considered as the regions of slow moving fluids because of the high viscosity $\left(\mu_{y i e l d} \geq 10^{3} \mu\right)$ in the context of bi-viscosity regularisation. The shapes and sizes of AURs are dependent on the choice of $\mu_{\text {yield }}$ [11-18] but the qualitative and quantitative distributions of stream function and isotherms remain independent of the value of $\mu_{\text {yield }}$ for $\mu_{\text {yield }} \geq 10^{3} \mu$, and thus a fixed value $\mu_{y i e l d}=10^{4} \mu$ is used here for all the simulations for the sake of highfidelity. The precise shapes and sizes of AURs do not affect the mean Nusselt number in the present study. The size of the AURs increases, and thermal transport becomes increasingly conduction-dominated as the value of $B n$ increases. Fluid flow practically stops for large values of $B n$ and thermal transport takes place purely due to thermal conduction (see Fig. 2). The relative contribution of convection to conduction on the overall thermal transport can be quantified in terms of the mean Nusselt number. For example, $\overline{N u}_{c y}=1.0$ in this configuration indicates that the thermal transport is principally conduction-driven, whereas $\overline{N u}_{c y}>1.0$ suggests that convection influences the thermal transport, and the strengthening of convection in comparison to thermal conduction is reflected in the increase of the mean Nusselt number $\overline{N u}_{c y}$. It will be shown later that $\overline{N u}_{c y}$ indeed decreases with increasing $B n$ due to weakening of convection and settles to $\overline{N u}_{c y}=1.0$ for large values of Bingham number (see Fig. 6 later in the paper).

Although Fig. 2 shows the results only for the CWHF boundary condition, the distributions of $\theta$ and stream function $\Psi$ for the CWT boundary condition remain qualitatively similar to those obtained for the CWHF boundary condition, and thus are not explicitly shown here for the sake of conciseness. Furthermore, the effects of $B n$ on the distributions of $\theta$ and stream function $\Psi$ for the CWT boundary condition are qualitatively similar to those in the case of CWHF boundary condition. This is consistent with previous findings by Turan et al. [14] who reported 
qualitatively similar behaviour for laminar Rayleigh-Bénard convection of Bingham fluids in square enclosures (i.e. $r_{i} \rightarrow \infty$ ) for both CWT and CWHF boundary conditions.

\section{Effects of $r_{i} / L$ and $R a$}

The variations of $\theta$ and $W$ with $\left(r-r_{i}\right) / L$ at $z / L=0.5$ for different values of $r_{i} / L$ at $R a=10^{4}$, $10^{5}$ and $\operatorname{Pr}=500$ are shown in Fig. 3 for $B n=0$ and $B n=0.03$ case. The corresponding variation of $U$ with $z / L$ at $0.5\left(r_{i}+r_{o}\right) / L$ for $B n=0$ and $B n=0.03$ is shown in Fig. 3 for both CWT and CWHF boundary conditions at $R a=10^{4}, 10^{5}$ and $P r=500$. It can be seen from Fig. 3 that the temperature difference between hot and cold walls is smaller in the CWHF configuration than that for the CWT boundary condition for a given set of values of $R a, P r, B n$. For the CWHF boundary condition, the wall heat flux can be estimated as $q \sim k \Delta T / \delta_{t h}$ where $\Delta T$ and $\delta_{t h}$ are the characteristic temperature difference and the thickness of thermal boundary layer on horizontal walls, respectively. This suggests that the non-dimensional temperature $\theta$ in the case of CWHF boundary condition scales as $\theta \sim \Delta T k / q L \sim q \delta_{t h} k / q L k \sim O\left(\delta_{t h} / L\right)$, whereas $\theta \sim O(1)$ for the CWT boundary condition. The above discussion suggests that $\theta \sim O\left(\delta_{t h} / L\right)$ for the CWHF boundary condition is expected to increase with increasing $\delta_{t h}$, as the thermal boundary layer thickness increases with an increase in Bingham number due to the weakening of convection strength (see Fig. 3). The convection strength also weakens with decreasing $R a$, which is reflected in the thickening of thermal boundary layer thickness. Thus, the temperature difference between horizontal walls also increases with decreasing $R a$ for the CWHF boundary condition. Higher temperature difference between the horizontal walls leads to stronger convection in the CWT configuration than in the corresponding CWHF configuration for the same set of values of $r_{i} / L, R a, \operatorname{Pr}$ and $B n$. This can be verified from Fig. 3 which shows that the magnitudes of $U$ and $W$ are greater in the CWT configuration than in the CWHF configuration for a given set of values of $r_{i} / L, R a, \operatorname{Pr}$ and $B n$. 
Figure 3 further shows that $r_{i} / L$ does not significantly affect the temperature difference between horizontal walls for both $B n=0$ and $B n=0.03$ regardless of the boundary condition at low nominal $R a$ values (i.e. $R a=10^{4}$ ). However, the temperature difference between horizontal walls is significantly influenced by $r_{i} / L$ for high values of $R a$ (i.e. $R a=10^{5}$ ) for $r_{i} / L<1$ for both CWT and CWHF boundary conditions. This behaviour arises due to a change in the streamline pattern for $r_{i} / L<1$ and $R a \geq 5 \times 10^{4}$ (see in Fig. 4), which leads to a modification of temperature distribution between hot and cold walls regardless of the boundary condition. It is worth noting that the magnitudes of $U$ and $W$ for $R a=10^{5}$ are greater than the corresponding values for $R a=10^{4}$ due to strengthening of buoyancy forces with increasing $R a$ for both CWT and CWHF configuration.

It can further be seen from Fig. 3 that non-dimensional axial velocity component $W$ decreases while the non-dimensional radial velocity component $U$ increases with increasing $r_{i} / L$ for both $B n=0$ and $B n=0.03$ cases. Additionally, the magnitudes of $W$ and $U$ are smaller in the Bingham fluid cases than in the corresponding Newtonian fluid cases for a given set of values of nominal Rayleigh and Prandtl numbers.

One obtains $w \sim \sqrt{g \beta\left(T_{H}-T_{C}\right) L}\left(w \sim \sqrt{g \beta q \delta_{t h} L / k}\right)$ by equating the order of magnitudes of inertial and buoyancy terms for the CWT (CWHF) boundary condition. Alternatively, these scaling can be expressed as: $w \sim(\alpha / L) \sqrt{\operatorname{RaPr}}\left(w \sim(\alpha / L) \sqrt{\operatorname{RaPr}\left(\delta_{t h} / L\right)}\right)$ for the CWT (CWHF) boundary condition, which suggests that the magnitude of $W$ is expected to be greater in the CWT configuration than in the case of CWHF boundary condition for a given set of values of $R a$ and $\operatorname{Pr}$ (because $\delta_{t h} \ll L$ in boundary layer transport). Based on the continuity equation for axisymmetric geometry one obtains: 
$\frac{1}{r} \frac{\partial(r u)}{\partial r} \sim\left(\frac{u}{r}+\frac{u}{L}\right) \sim \frac{\partial w}{\partial z} \sim \frac{w}{L}$

which leads to the following relation based on scaling estimate of $u$ :

$u \sim \frac{w r}{(r+L)} \sim \frac{w}{\left(1+L / r_{i}\right)} \sim \frac{\alpha}{L} \frac{\sqrt{R a P r}}{\left(1+L / r_{i}\right)} \quad$ for $\quad \mathrm{CWT}$

$u \sim \frac{w r}{(r+L)} \sim \frac{w}{\left(1+L / r_{i}\right)} \sim \frac{\alpha}{L} \frac{\sqrt{R a P r}}{\left(1+L / r_{i}\right)} \sqrt{\frac{\delta_{t h}}{L}}$ for $\mathrm{CWHF}$

As $\delta_{t h} / L<1$, the magnitude of $U$ is expected to be greater in the CWT configuration than in the case of CWHF boundary condition for a given set of values of $\operatorname{Ra}$ and $\operatorname{Pr}$ (because $\delta_{t h} \ll$ $L)$. According to Eq. 16 (i.e. $u \sim w /\left(1+L / r_{i}\right)$ ) the magnitude of the radial velocity component $u$ increases with increasing $r_{i} / L$ which is consistent with numerical findings for both Newtonian and Bingham fluid cases as shown in Fig. 3. The contours of non-dimensional temperature $\theta$ and non-dimensional stream function $\Psi$ with AURs (shown in grey) for $B n=0$ (i.e. Newtonian fluid) and $B n=0.15$ fluid cases for different values of $r_{i} / L$ for $R a=10^{5}$ and $\operatorname{Pr}=500$ are shown in Fig. 4 for the CWT boundary condition. It is evident from the Fig. 4 that the flow pattern (i.e. number of cells/rolls) changes for $r_{i} / L \leq 1$ for Newtonian fluids However, this flow pattern reverts to the one cell structure with increasing $B n$.

Following previous analyses [11-18] a scaling analysis can be performed to elucidate the expected behaviours in response to the changes in key parameters (e.g. $r_{i} / L, R a$ and $B n$ ). One obtains the following expression by equating the order of magnitudes of inertial and viscous terms in the radial direction:

$\rho \frac{u^{2}}{L} \sim \frac{1}{\delta_{1}}\left(\tau_{y}+\mu \frac{u}{\delta_{1}}\right)$

Using Eqs. 16i and $16 \mathrm{ii}$ in eq. $17 \mathrm{i}$ leads to:

$\delta_{1} \sim L\left[\sqrt{\left(\frac{B n}{2}\right)^{2} \frac{P r}{R a}\left(1+L / r_{i}\right)^{4}+\sqrt{\frac{P r\left(1+L / r_{i}\right)^{2}}{R a}}}+\left(\frac{B n}{2}\right) \sqrt{\frac{P r}{R a}}\left(1+L / r_{i}\right)^{2}\right]$ for CWT 


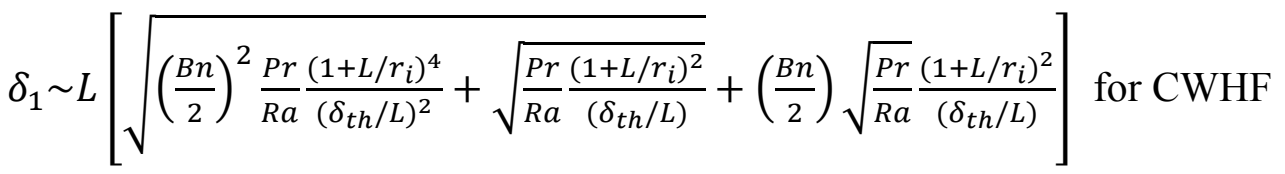

where $\delta_{1}$ is the hydrodynamic boundary layer thickness on the horizontal wall. Similarly, the hydro-dynamic boundary layer thickness on the vertical wall $\delta$ can be scaled in the following manner by equating the magnitudes of inertial and viscous terms in the vertical direction (i.e. $\left.\rho w^{2} / L \sim\left(\tau_{y}+\mu w / \delta\right) / \delta\right)[13,14]:$

$\delta \sim L\left[\sqrt{\left(\frac{B n}{2}\right)^{2} \frac{P r}{R a}+\sqrt{\frac{P r}{R a}}}+\left(\frac{B n}{2}\right) \sqrt{\frac{P r}{R a}}\right]$ for CWT

$\delta \sim L\left[\sqrt{\left(\frac{B n}{2}\right)^{2} \frac{P r}{R a} \frac{1}{\left(\delta_{t h} / L\right)^{2}}+\sqrt{\frac{P r}{R a} \frac{1}{\left(\delta_{t h} / L\right)}}}+\left(\frac{B n}{2}\right) \sqrt{\frac{P r}{R a}} \frac{1}{\left(\delta_{t h} / L\right)}\right]$ for CWHF

Equations 17 and 18 indicate that the boundary layer thickness increases with decreasing (increasing) nominal Rayleigh number $R a$ (nominal Bingham number $B n$ ). It is worth noting that Eq. 17 approaches Eq. 18 for square enclosures (i.e. $r_{i} / L \rightarrow \infty$ ), and Eq. 18 was previously obtained for square enclosures by Turan et al. $[13,14]$.

Based on the scaling estimates in Eqs. 17 and18, it is possible to estimate the effective viscosity in both vertical and horizontal boundary layers (i.e. $\mu_{e f f}{ }^{V}$ and $\mu_{e f f}{ }^{H}$ ) in the following manner $\left(\mu_{e f f}{ }^{V} \sim \mu+\tau_{y} \delta / w\right.$ and $\left.\mu_{e f f}{ }^{H} \sim \mu+\tau_{y} \delta_{1} / u\right)$ :

For CWT configuration [13]

$$
\begin{aligned}
& \frac{\mu_{e f f} V}{\mu} \sim 1+B n\left[\sqrt{\left(\frac{B n}{2}\right)^{2} \frac{P r}{R a}+\sqrt{\frac{P r}{R a}}}+\left(\frac{B n}{2}\right) \sqrt{\frac{P r}{R a}}\right] \\
& \frac{\mu_{e f f}{ }^{H}}{\mu} \sim 1+\frac{B n}{1+L / r_{i}}\left[\sqrt{\left(\frac{B n}{2}\right)^{2} \frac{P r}{R a}\left(1+L / r_{i}\right)^{4}+\sqrt{\frac{P r\left(1+L / r_{i}\right)^{2}}{R a}}}+\left(\frac{B n}{2}\right) \sqrt{\frac{P r}{R a}}\left(1+L / r_{i}\right)^{2}\right]
\end{aligned}
$$

For CWHF configuration $[13,14]$ 
$\frac{\mu_{e f f} V}{\mu} \sim 1+B n \sqrt{\frac{L}{\delta_{t h}}}\left[\sqrt{\left(\frac{B n}{2}\right)^{2} \frac{P r}{R a} \frac{1}{\left(\delta_{t h} / L\right)^{2}}+\sqrt{\frac{P r}{R a} \frac{1}{\left(\delta_{t h} / L\right)}}}+\left(\frac{B n}{2}\right) \sqrt{\frac{P r}{R a}} \frac{1}{\left(\delta_{t h} / L\right)}\right]$

$\frac{\mu_{e f f}{ }^{H}}{\mu} \sim 1+\frac{B n}{1+L / r_{i}} \sqrt{\frac{L}{\delta_{t h}}}\left[\sqrt{\left(\frac{B n}{2}\right)^{2} \frac{P r}{R a} \frac{\left(1+L / r_{i}\right)^{4}}{\left(\delta_{t h} / L\right)^{2}}+\sqrt{\frac{P r}{R a} \frac{\left(1+L / r_{i}\right)^{2}}{\left(\delta_{t h} / L\right)}}}+\left(\frac{B n}{2}\right) \sqrt{\frac{P r}{R a}} \frac{\left(1+L / r_{i}\right)^{2}}{\left(\delta_{t h} / L\right)}\right]$

Using Eqs. 19 and 20, the effective Rayleigh numbers (i.e. $R a_{e f f}{ }^{V}$ and $R a_{e f f}{ }^{H}$ ) in both vertical and horizontal boundary layers can be estimated in the following manner:

For CWT configuration (i.e. $\left.R a_{e f f}=\rho g \beta\left(T_{H}-T_{C}\right) L^{3} / \mu_{e f f} \alpha\right)$ :

$$
\begin{aligned}
& R a_{e f f}{ }^{V} \sim R a /\left\{1+B n\left[\sqrt{\left(\frac{B n}{2}\right)^{2} \frac{P r}{R a}+\sqrt{\frac{P r}{R a}}}+\left(\frac{B n}{2}\right) \sqrt{\frac{P r}{R a}}\right]\right\} \\
& R a_{e f f}^{H} \sim R a /\left\{1+\frac{B n}{1+L / r_{i}}\left[\sqrt{\left(\frac{B n}{2}\right)^{2} \frac{P r}{R a}\left(1+L / r_{i}\right)^{4}+\sqrt{\frac{P r\left(1+L / r_{i}\right)^{2}}{R a}}}+\left(\frac{B n}{2}\right) \sqrt{\frac{P r}{R a}}\left(1+L / r_{i}\right)^{2}\right]\right\}
\end{aligned}
$$

For CWHF configuration (i.e. $R a_{e f f}=\rho g \beta q L^{4} / k \mu_{e f f} \alpha$ ):

$$
\begin{aligned}
& R a_{e f f}{ }^{V} \sim R a /\left\{1+B n \sqrt{\frac{L}{\delta_{t h}}}\left[\sqrt{\left(\frac{B n}{2}\right)^{2} \frac{P r}{R a} \frac{1}{\left(\delta_{t h} / L\right)^{2}}+\sqrt{\frac{P r}{R a} \frac{1}{\left(\delta_{t h} / L\right)}}}+\left(\frac{B n}{2}\right) \sqrt{\frac{P r}{R a}} \frac{1}{\left(\delta_{t h} / L\right)}\right]\right\} \\
& R a_{e f f}{ }^{H} \sim R a /\left\{1+\frac{B n}{1+L / r_{i}} \sqrt{\frac{L}{\delta_{t h}}}\left[\sqrt{\left.\left.\left(\frac{B n}{2}\right)^{2} \frac{P r}{R a} \frac{\left(1+L / r_{i}\right)^{4}}{\left(\delta_{t h} / L\right)^{2}}+\sqrt{\frac{P r}{R a} \frac{\left(1+L / r_{i}\right)^{2}}{\left(\delta_{t h} / L\right)}}+\left(\frac{B n}{2}\right) \sqrt{\frac{P r}{R a}} \frac{\left(1+L / r_{i}\right)^{2}}{\left(\delta_{t h} / L\right)}\right]\right\}}\right.\right.
\end{aligned}
$$

Equations 21 and 22 indicate that the effective Rayleigh number remains smaller than the nominal Rayleigh number and the effective Rayleigh number decreases with increasing $B n$ for both CWT and CWHF boundary conditions. Furthermore, the effective Rayleigh number for the CWHF boundary condition is smaller than the CWT boundary condition for same set of values of $R a, \operatorname{Pr}$ and $B n$ because $\delta_{t h}$ is expected to be smaller than $L$. The scaling estimates for $R a_{e f f}{ }^{V}$ (i.e. eqs. $21 \mathrm{i}$ and 22i) are consistent with previous findings in the context of square enclosures. 
Equations 19 and 20 indicate that $\delta_{1}>\delta$ and $u<w$, thus $\mu_{e f f}{ }^{H}$ is expected to be greater than $\mu_{e f f}{ }^{V}$ in cylindrical enclosures. This further implies that $R a_{e f f}{ }^{H}$ is expected to be smaller than $R a_{e f f}{ }^{V}$ in cylindrical enclosures as it is shown in Eqs. 21 and 22. However, they approach each other for $r_{i} / L \rightarrow \infty$ (i.e. in the limit of square enclosures). It is worth noting that the velocity magnitudes in both vertical and horizontal directions remain of the same order for a twodimensional square enclosure, which leads to $\delta \sim \delta_{1}$ and $R a_{\text {eff }}{ }^{V} \sim R a_{e f f}{ }^{H}$.

\section{Behavior on mean Nusselt number of cylindrical enclosures $\overline{N u}_{c y}$}

The variation of $\overline{N u}_{c y}$ with $r_{i} / L$ for different $R a$ is shown in Fig. 5 for Newtonian fluids (Bn $=0)$ at $P r=500 .^{2}$ It can be seen from Fig. 5 that $\overline{N u}_{c y}$ increases with increasing $R a$ but remains insensitive to the changes in $r_{i} / L$ for $r_{i} / L>1$ in the nominal Rayleigh number range for both CWT and CWHF boundary conditions. However, $\overline{N u}_{c y}$ increases sharply with increasing $r_{i} / L$ for $r_{i} / L<1$ in the case of $R a=10^{5}$. This behaviour is particularly prevalent in the CWT case. This can be explained by the change in the flow pattern, as shown in Figs. 4-5. The changes in the flow patterns for $R a \geq 5 \times 10^{4}$ and $r_{i} / L \leq 1$ modify the distributions of isotherms between hot and cold walls, which is reflected in the sharp increase in $\overline{N u}_{c y}$ (see Figs. 4-5).

The wall heat flux $q \sim h \Delta T$ can be scaled as: $q \sim h \Delta T \sim k \Delta T / \delta_{t h} \sim k\left(\Delta T / \delta_{1}\right) f_{2}$ [11-18], so the mean Nusselt number can be scaled as: $\overline{N u} \sim h L / k \sim q L / \Delta T k \sim L / \delta_{t h} \sim\left(L / \delta_{1}\right) f_{2}$, where $f_{2}\left(\operatorname{Ra}, \operatorname{Pr}, r_{i} / L, B n\right)$ is a function which accounts for the ratio of hydro-dynamic and thermal boundary layer thicknesses (i.e. $\left.f_{2} \sim \delta_{1} / \delta_{t h}\right)$. However, the exact form of $f_{2}\left(R a, P r, r_{i} / L, B n\right)$ is not important for the following discussion. Using Eqs. 17ii and 17iii it is possible to estimate

${ }^{2}$ The mean Nusselt number $\overline{N u}_{c y}$ from computational simulation results is evaluated using eq. 9 for Figs. 5, 6 and 8. 
the mean Nusselt number for natural convection of Newtonian fluids by putting $B n=0$ as: $\overline{N u}_{c y} \sim(R a / P r)^{0.25} f_{2}(R a, P r) /\left(1+L / r_{i}\right)^{0.5}\left(\overline{N u}_{c y} \sim(R a / P r)^{0.2}\left\{f_{2}(R a, P r)\right\}^{0.8} /\left(1+L / r_{i}\right)^{0.4}\right)$ for the CWT (CWHF) boundary condition. These scalings suggest that $\overline{N u}_{c y}$ is expected to increase with increasing $R a$ and $r_{i} / L$, which are consistent with the observations made from Fig. 5. Furthermore, one obtains the mean Nusselt number estimates for square enclosures $\overline{N u}_{s q} \quad$ by using $B n=0 \quad$ and $\quad L / r_{i}=0 \quad$ as: $\quad \overline{N u}_{s q} \sim(R a / P r)^{0.25} f_{2}(R a, P r)$ $\left(\overline{N u}_{s q} \sim(R a / P r)^{0.2}\left\{f_{2}(R a, P r)\right\}^{0.8}\right)$ for the CWT (CWHF) boundary condition. The following correlations for the mean Nusselt number were proposed in $[13,14]$ for natural convection of Newtonian fluids in square enclosures $\overline{N u}_{s q}$ :

$\overline{N u}_{s q}=0.178 R a^{0.269}[\operatorname{Pr} /(1+\operatorname{Pr})]^{0.02}$ for CWT

$\overline{N u}_{s q}=0.289 R a^{0.214}[\operatorname{Pr} /(1+\operatorname{Pr})]^{0.017}$ for CWHF

Equations $23 \mathrm{i}$ and $23 \mathrm{ii}$ are consistent with the scaling estimates $\overline{N u}_{s q} \sim(R a / P r)^{0.25} f_{2}(R a, P r)$ and $\overline{N u}_{s q} \sim(R a / P r)^{0.2}\left\{f_{2}(R a, P r)\right\}^{0.8}$, respectively. A comparison between $\overline{N u}_{s q}=$ $0.178 R a^{0.269}[\operatorname{Pr} /(1+\operatorname{Pr})]^{0.02} \quad$ (or $\quad \overline{N u}_{s q}=0.289 \operatorname{Ra}^{0.214}[\operatorname{Pr} /(1+\operatorname{Pr})]^{0.017}$ ) and $\overline{N u}_{s q} \sim(R a / P r)^{0.25} f_{2}(R a, P r) \quad\left(\right.$ or $\left.\overline{N u}_{s q} \sim(R a / P r)^{0.2}\left\{f_{2}(R a, P r)\right\}^{0.8}\right)$ reveals that $f_{2}=$ $0.178 R a^{0.019} \operatorname{Pr}^{0.27} /(1+\operatorname{Pr})^{0.02}$ (or $f_{2}=0.212 R a^{0.0175} \operatorname{Pr}^{0.27} /(1+P r)^{0.02}$ ) for the CWT (or CWHF) boundary condition. The predictions of Eq. 23 are shown in Fig. 5, which indicates that $\overline{N u}_{c y}$ can be satisfactorily predicted with the help of Eq. 23 for all cases considered here except for $R a=10^{5}$ and $r_{i} / L \leq 1$. Equation 23 is valid for the flow pattern with one cell within the enclosure but two cells have been found for $R a=10^{5}$ and $r_{i} / L \leq 1$, which lead to a reduction in $\overline{N u}_{c y}$ in comparison to the values obtained for $r_{i} / L>1$. The variation of the mean Nusselt number $\overline{N u}_{c y}$ with $B n$ for different values of $r_{i} / L$ at $R a=10^{5}$ and $\operatorname{Pr}=500$ for both CWT (top) and CWHF (bottom) boundary conditions is shown in Fig. 6 for Bingham fluids. It is evident from Fig 6 that the $\overline{N u}_{c y}$ decreases with increasing $B n$ which is indicative of the 
weakening of convective transport due to strengthening of additional flow resistance arising from yield stress. Furthermore, Fig. 6 further shows that $\overline{N u}_{c y}$ settles to unity once a threshold value of Bingham number $\left(B n_{\max }\right)_{c y}$ is surpassed. Here $\left(B n_{\max }\right)_{c y}$ is taken to be the nominal Bingham number where $\overline{N u}_{c y}=1.01$, so that $\overline{N u}_{c y}<1.01$ for $B n>\left(B n_{\max }\right)_{c y}$. The fluid becomes totally un-yielded for $B n>\left(B n_{\max }\right)_{c y}$, and the thermal transport takes place purely due to conduction under this situation. Thus, there is no point in simulating cases with $\mathrm{Bn}>$ $\left(B n_{\max }\right)_{c y}$ because the heat transfer remains purely conduction-driven and $\overline{N u}_{c y}$ remains unity. The range of Bingham number (i.e. $0<B n<\left(B n_{\max }\right)_{c y}$ ) depends on $R a$ and $r_{i} / L$ for a given value of $\operatorname{Pr}$. During the course of this analysis, the simulations have been conducted by increasing (decreasing) $B n$ until $\left(B n_{\max }\right)_{c y}(B n=0)$ is reached from $B n=0(B n=$ $\left.\left(B n_{\max }\right)_{c y}\right)$ in order to assess if the variation of $\overline{N u}_{c y}$ with $B n$ shows any hysteresis. It has been found that hysteresis occurs (i.e. $\overline{N u}_{c y}$ is indeed multi-valued for a given value of $B n$ and different flow patterns are obtained for a given value of $B n$ ) for $R a \geq 5 \times 10^{4}$ and $r_{i} / L \leq 1$ depending on the starting condition (see Fig.6) for both CWT and CWHF conditions. By contrast, starting condition has no influence on the results for $r_{i} / L>1$ for a given value of $R a$ for both CWT and CWHF configurations.

The variation of $\left(B n_{\max }\right)_{c y}$ with $r_{i} / L$ for different values of $R a$ at $P r=500$ for both CWT and CWHF boundary conditions is shown in Fig. 7. It can be seen from Fig. 7 that $\left(B n_{\max }\right)_{c y}$ increases with increasing $R a$. The effects of buoyancy force strengthen with increasing $R a$ and thus convection could be sustained up to high values of Bingham number for large values of nominal Rayleigh number. This is reflected in the increasing trend of $\left(B n_{\max }\right)_{c y}$ with increasing $R a$. Figure 7 further shows that $\left(B n_{\max }\right)_{c y}$ for a given value of $R a$ for the CWT boundary condition is greater than in the case of CWHF boundary condition due to stronger 
convection in the CWT configuration as a result of higher temperature difference between active walls (see Fig. 3). Figure 7 shows that the $\left(B n_{\max }\right)_{c y}$ increases with increasing $r_{i} / L$ before approaching the corresponding value for a square enclosure $\left(B n_{\max }\right)_{s q}$ in the limit of $r_{i} / L \rightarrow \infty$. This indicates that although $\overline{N u}_{c y}$ remains independent of $r_{i} / L$ for Newtonian fluids $(B n=0)$ for $r_{i} / L>1$, convection strengthens with increasing $r_{i} / L$ for Bingham fluids for both CWT and CWHF boundary conditions.

The quantity $\left(B n_{\max }\right)_{c y}$ can be estimated for the CWT boundary condition by considering $\overline{N u}_{c y} \sim L / \delta_{t h} \sim L f_{2} / \delta_{1} \sim O(1)$. This along with Eq. 17ii yields for the CWT boundary condition:

$$
\left(B n_{\max }\right)_{c y} \sim \frac{f_{2} \sqrt{R a / P r}}{\left(1+L / r_{i}\right)^{2}}-\frac{1}{\left(1+L / r_{i}\right) f_{2}}
$$

Using $r_{i} / L \rightarrow \infty$ in Eq. 24i yields the estimate of the corresponding threshold value of Bingham number for square enclosures for the CWT boundary condition:

$$
\left(B n_{\max }\right)_{s q} \sim f_{2} \sqrt{R a / P r}-\frac{1}{f_{2}}
$$

It can be seen from Eq. $24 \mathrm{i}$ that $\left(B n_{\max }\right)_{c y}$ is expected to increase with increasing $R a$ and $r_{i} / L$, which are consistent with the observations made from Fig. 7. Although $\delta_{1}$ and $\left(B n_{\max }\right)_{c y}$ cannot be analytically obtained from Eq. 17iii, the same qualitative behaviour as that of the CWT boundary condition can be expected for the CWHF boundary condition.

The quantity $\left(B n_{\max }\right)_{s q}$ is parameterised in [17] in the following manner for $10 \leq \operatorname{Pr} \leq 500$ :

$$
\begin{aligned}
& \left(B n_{\text {max }}\right)_{s q}=\left[\left(\frac{0.00048 P r+0.132}{P r+18.15}\right) R a^{0.525}\right]-\frac{0.0389 P r+7.687}{P r+13.56} \text { for CWT } \\
& \left(B n_{\max }\right)_{s q}=\left[\left(\frac{P r+236}{2707 P r+40280}\right) R a^{0.525}\right]-\frac{P r+128}{44.6 P r+271} \text { for CWHF }
\end{aligned}
$$


Equation 24iii is consistent with the scaling estimate presented in Eq. 24ii. Using Eq. 24, $\left(B n_{\max }\right)_{c y}$ is correlated here in the following manner:

$\left(B n_{\text {max }}\right)_{c y}=\left(B n_{\max }\right)_{s q} /\left[1+a_{o}\left(L / r_{i}\right)^{b_{o}}\right]$

where $a_{o}=0.0127 R a^{0.118}$ and $b_{o}=0.142 R a^{0.125}$ for CWT

$a_{o}=0.05$ and $b_{o}=0.292 R a^{0.077}$ for $\mathrm{CWHF}$

The predictions of Eq. 25 are shown in Fig. 7 which shows this correlation satisfactorily predicts $\left(\mathrm{R}^{2}=0.99\right)\left(B n_{\max }\right)_{c y}$ for Bingham fluids in square cross-sectioned cylindrical enclosures for $10^{3} \leq R a \leq 10^{5}, 0.125 \leq r_{i} / L \leq 16$ at $\operatorname{Pr}=500$. It is worth noting that $\left(B n_{\max }\right)_{c y}$ becomes equal to $\left(B n_{\max }\right)_{s q}$ in the limit of $r_{i} / L \rightarrow \infty$ which ensures that asymptotic condition $\left(B n_{\max }\right)_{c y}=\left(B n_{\max }\right)_{s q}$ for $r_{i} / L \rightarrow \infty$ is satisfied.

Using Eq. 17ii one obtains the following estimate of mean Nusselt number $\overline{N u}_{c y} \sim\left(L / \delta_{1}\right) f_{2}$ for the CWT boundary condition:

$$
\overline{N u}_{c y} \sim f_{2}\left[\sqrt{\left(\frac{B n}{2}\right)^{2} \frac{P r}{R a}\left(1+L / r_{i}\right)^{4}+\sqrt{\frac{P r\left(1+L / r_{i}\right)^{2}}{R a}}}+\left(\frac{B n}{2}\right) \sqrt{\frac{P r}{R a}}\left(1+\frac{L}{r_{i}}\right)^{2}\right]^{-1}
$$

Equation $26 \mathrm{i}$ indicates that $\overline{N u}_{c y}$ is expected to increase with increasing $R a$ and $r_{i} / L$, and decrease with increasing $B n$. It is not possible to obtain an analytical scaling estimate of $\overline{N u}_{c y}$ from Eq. 17iii but the mean Nusselt number behaviour in response to $R a, B n$ and $r_{i} / L$ for CWHF boundary condition is expected to be qualitatively similar to that in the case of CWT boundary condition. A correlation for $\overline{N u}_{s q}$ was proposed in [13] for natural convection of Bingham fluids in square enclosures in the range $0 \leq B n \leq\left(B n_{\max }\right)_{s q}$. The methodology of [13] has been utilised here along with Eq. $26 \mathrm{i}$ and $\left(\overline{N u}_{c y}\right)_{B n=0} \sim(R a / P r)^{0.25} f_{2}(R a, P r) /$ $\left(1+L / r_{i}\right)^{0.5}$ to propose a correlation of $\overline{N u}_{c y}$ for $0 \leq B n \leq\left(B n_{\max }\right)_{c y}$ in the following manner for the CWT boundary condition: 
$\frac{\overline{N u}_{c y}-1}{\left(\overline{N u}_{c y}\right)_{B n=0}-1}=\frac{2\left[1-\left(B n^{*} / B n_{m a x}^{*}\right)^{b}\right]^{c}}{B n^{*}+\sqrt{B n^{* 2}+4}}$ when $\left(\overline{N u}_{c y}\right)_{B n=0}>1$

$\overline{N u}_{c y}=1$ when $\left(\overline{N u}_{c y}\right)_{B n=0}=1$

where $B n_{\max }^{*}=B n_{\max }\left(1+L / r_{i}\right)^{1.5}(\mathrm{Ra} / \mathrm{Pr})^{-1 / 4}, b$ and $c$ are the correlation parameters. Following Ref. [13], the functional forms given by Eqs. 26ii and 26iii are taken to be valid also for the CWHF boundary condition. Turan et al. $[13,14]$ proposed $b_{s q}=0.6$ and $c_{s q}=$ $0.025 \operatorname{Ra}^{0.171} \operatorname{Pr}^{0.095}\left(b_{s q}=0.75\right.$ and $\left.c_{s q}=0.0818 R a^{0.0191} \operatorname{Pr}^{0.054}\right)$ for the CWT (CWHF) boundary condition $[13,14]$. Accordingly in the current study $b$ and $c$ have been parameterized in the range of $2 \leq r_{i} / L \leq 16\left(0.25 \leq r_{i} / L \leq 16\right)$ as:

$b_{c y}=b_{s q}$ and $c_{c y}=c_{s q} /\left[1+m_{o}\left(L / r_{i}\right)^{n_{o}}\right]$

$m_{o}=4.616-0.385 \ln R a$ and $n_{o}=0.2$ for $2 \leq r_{i} / L \leq 16$ for CWT

$m_{o}=0.1$ and $n_{o}=0.021 R a^{0.274}$ for $0.25 \leq r_{i} / L \leq 16$ for CWHF

The predictions of Eqs. 26ii and iii are shown in Fig. 8 which shows that this correlation satisfactorily predicts $\left(\mathrm{R}^{2}=0.99\right.$ and $3 \%$ maximum percentage error) $\overline{N u}_{c y}$ for natural convection of Bingham fluids in square-cross sectioned cylindrical enclosures. It is also worth noting that $(c)_{c y}$ becomes equal to $(c)_{s q}$ in the limit of $r_{i} / L \rightarrow \infty$ which ensures that asymptotic condition $\overline{N u}_{c y}=\overline{N u}_{s q}$ for $r_{i} / L \rightarrow \infty$ is satisfied in Eqs. 26ii-iii. Note that the correlation given by Eqs. 26ii-iii is only valid for the range of $r_{i} / L$ where one cell convection pattern is observed. Therefore, the values corresponding to $r_{i} / L=0.25$ and 1.0 for the CWT boundary condition are not shown in Fig. 8 since multiple cell patterns are obtained under these conditions, and Eqs. 26ii-iii are valid for the range of $2 \leq r_{i} / L \leq 16\left(0.25 \leq r_{i} / L \leq\right.$ 16) for the CWT (CWHF) boundary condition.

Turan et al. $[13,14]$ analysed the influences of $\operatorname{Pr}$ for the range of $0.1 \leq \operatorname{Pr} \leq 100$ on $\overline{N u}_{s q}$ for laminar Rayleigh-Bénard convection of Bingham fluids in square enclosures (i.e. $r_{i} \rightarrow \infty$ ) 
for both CWT and CWHF boundary conditions. It has been found that the $\overline{N u}_{s q}$ decreases with increasing $\operatorname{Pr}$ for large values of $B n$ values whereas, $\overline{N u}_{s q}$ increases with increasing $\operatorname{Pr}$ for small values of $B n$ values for both CWT and CWHF boundary conditions. Furthermore, $B n_{\max }$ decreases with increasing $\operatorname{Pr}[13,14]$. Interested readers are requested to refer to Refs. $[13,14]$ for detailed information in this regard. As the functional relations given by Eqs. 26ii and 26 iii are valid for natural convection of Bingham fluids in annual enclosures irrespective of the value of $\mathrm{Pr}$, and the parameterisation of $B n_{\max }$ is proposed here based on the expressions originally proposed by Yigit et al. [17] for $10 \leq \operatorname{Pr} \leq 500$, it can be expected the correlation of $\overline{N u}_{c y}$ given by Eqs. 26ii-iii likely to valid for a range of different Prandtl numbers and a limited number of simulations have been carried out for $\operatorname{Pr}=100$ and Eqs. 26ii-iii have indeed been found to predict $\overline{N u}_{c y}$ satisfactorily also for these cases.

\section{CONCLUSIONS}

Steady-state laminar Rayleigh-Bénard convection of Bingham fluids in cylindrical annual enclosures has been analysed for the range of nominal Rayleigh number $10^{3} \leq R a \leq 10^{5}$ at a representative value of nominal Prandtl number (i.e. $\mathrm{Pr}=500$ ) for different values of normalised inner radius $0 \leq r_{i} / L \leq 16$ for both constant wall temperature and constant wall heat flux boundary conditions. It has been found that the buoyancy-driven transport strengthens with increasing nominal Rayleigh number $R a$ for both Newtonian and Bingham fluids but the mean Nusselt number $\overline{N u}_{c y}$ for Bingham fluids has been found to be smaller than the corresponding values obtained for Newtonian fluids for both CWT and CWHF boundary conditions due to additional flow resistance arising from yield stress in the case of Bingham fluids. Moreover, $\overline{N u}_{c y}$ decreases with increasing $B n$ and it assumes a value equal to unity (i.e. $\overline{N u}_{c y}=1.0$ ) for large values of $B n$ which indicates that thermal transport takes 
place purely due to thermal conduction as a result of high flow resistance arising from yield stress. Although the buoyancy force strengthens with increasing $R a$, the mean Nusselt number $\overline{N u}_{c y}$ does not show a monotonic increase with increasing $R a$ for small values of $r_{i} / L$. This behaviour arises due to the change in flow pattern (i.e. number of convection rolls/cells) for small values of $r_{i} / L$ for large values of $R a$. In contrast, only one cell flow pattern is obtained, and $\overline{N u}_{c y}$ monotonically increases with increasing $R a$ for large values of $r_{i} / L$. Furthermore, $\overline{N u}_{c y}$ has been found to increase with increasing $r_{i} / L$ but approaches the corresponding value obtained for square enclosures $\left(r_{i} \rightarrow \infty\right)$ for both CWT and CWHF boundary conditions for large values of $r_{i} / L$. It has also been found that flow pattern and the mean Nusselt number $\overline{N u}_{c y}$ are dependent on the starting conditions for Bingham fluid cases since hysteresis is evident for small values of $r_{i} / L$ for both CWT and CWHF boundary conditions. It has been found that convection could be sustained up to a higher value of Bingham number due to stronger convection arising from higher temperature difference between horizontal walls in the case of CWT boundary condition than in the case of CWHF configuration. Numerical findings have been used to propose a correlation for $\overline{N u}_{c y}$ in the range of $2 \leq r_{i} / L \leq 16$ for CWT and $0.25 \leq r_{i} / L \leq 16$ for CWHF boundary conditions. 


\section{REFERENCES}

[1] I. Catton, P.S. Ayyaswamy, and R.M. Clever, Natural convection flow in a finite, rectangular slot arbitrarily oriented with respect to the gravity vector, Int. J. Heat Mass Trans. 17 (1974) 173-184. Doi: 10.1016/0017-9310(74)90079-9

[2] S. Ostrach, Natural Convection in Enclosures, Journal of Heat Transfer 110 (1988) 11751190. Doi: $10.1115 / 1.3250619$

[3] M. A. Hassan, M. Pathak, M.K. Khan, Rayleigh-Bénard convection in Herschley-Bulkley fluid, J. Non-Newt. Fluid Mech. 226 (2015) 32-45. Doi:10.1016/j.jnnfm.2015.10.003

[4] M. Darbouli, C. Métivier, J.M. Piau, A. Magnin and A. Abdelali, Rayleigh-Bénard convection for viscoplastic fluids, Physics of Fluids 25 (2013) 023101. Doi: $10.1063 / 1.4790521$

[5] Z. Kebiche, C. Castelain, T. Burghelea, Experimental investigation of the Rayleigh-Bénard convection in a yield stress fluid, J. Non-Newt. Fluid Mech. 203 (2014) 9-23. Doi:10.1016/j.jnnfm.2013.10.005

[6] N.M. Wereley Magnetorheology: Advances and Applications, Royal Society of Chemistry, RSC Smart materials, Cambridge, UK, 2014.

[7] J. Zhang, D. Vola, I. A. Frigaard, Yield stress effects on Rayleigh-Bénard convection, J. Fluid Mech. 566 (2006) 389-419. Doi: 10.1017/S002211200600200X

[8] N. J. Balmforth and A.C. Rust, Weakly nonlinear viscoplastic convection, J. Non-Newt. Fluid Mech. 158 (2009) 36-45. Doi: 10.1016/j.jnnfm.2008.07.012 [9] A. Vikhansky, Thermal convection of a viscoplastic liquid with high Rayleigh and Bingham numbers, Phys. Fluids 21 (2009) 103103. Doi: 10.1063/1.3256166

[10] A. Vikhansky, On the onset of Bingham liquid in rectangular enclosures, J. Non-Newt. Fluid Mech. 165 (2010) 1713-1716. Doi: 10.1016/j.jnnfm.2010.09.003 
[11] O. Turan, N. Chakraborty, R.J. Poole, Laminar natural convection of Bingham fluids in a square enclosure with differentially heated side walls, J. Non-Newt. Fluid Mech. 165 (2010) 901-913. Doi: 10.1016/j.jnnfm.2010.04.013

[12] O. Turan, A. Sachdeva, R.J. Poole, N. Chakraborty, Laminar natural convection of Bingham fluids in a square enclosure with vertical walls subjected to constant heat flux, Num. Heat Trans. A 60 (2011) 381-409. Doi:10.1080/10407782.2011.594417

[13] O. Turan, A. Sachdeva, R.J. Poole, N. Chakraborty, Laminar Rayleigh-Bénard convection of yield stress fluids in a square enclosure, J. Non-Newt. Fluid Mech. 172 (2012) 83-96. doi:10.1016/j.jnnfm.2012.01.006

[14] O. Turan, R.J. Poole, N. Chakraborty, Boundary condition effects on natural convection of Bingham fluids in a square enclosure with differentially heated horizontal walls, J. Comput. Therm. Sci. 4 (2012) 77-97. Doi: 10.1615/ComputThermalScien.2012004759

[15] O. Turan, N. Chakraborty, R.J. Poole, “Aspect ratio effects in laminar natural convection of Bingham fluids in rectangular enclosures with differentially heated side walls, J. Non-Newt. Fluid Mech. 166 (2011) 208-230. Doi:10.1016/j.jnnfm.2010.12.002

[16] S. Yigit, R. J. Poole, N. Chakraborty, Laminar natural convection of Bingham fluids in inclined differentially heated square enclosures subjected to uniform wall temperatures, J. Heat Transfer Transactions of ASME 137 (2015) 052504. Doi:10.1115/1.4029763

[17] S. Yigit, R. J. Poole, N. Chakraborty, Effects of aspect ratio on natural convection of Bingham Fluids in rectangular enclosures with differentially heated horizontal walls heated from below, Int. J. Heat Mass Transfer 80 (2015) 727-736. Doi:10.1016/j.ijheatmasstransfer.2014.09.046

[18] O. Turan, R.J. Poole, N. Chakraborty, Influences of boundary conditions on laminar natural convection of Bingham fluids in rectangular enclosures with differentially heated side walls, Heat Trans. Eng. 35 (2014) 822-849. Doi:10.1080/01457632.2014.852870 
[19] M.A. Hassan, M. Pathak, M.K. Khan, Natural convection of viscoplastic fluids in a square enclosure, J. Heat Transfer Transactions of ASME 135 (2013) 122501.Doi: 10.1115/1.4024896 [20] H.A. Barnes, The yield stress — a review or ' $\pi \alpha \nu \tau \alpha \rho \varepsilon l$ ' - everything flows?, J Non-Newt. Fluid Mech. 81 (1999) 133-178. Doi: 10.1016/S0377-0257(98)00094-9

[21] E. J. O'Donovan, R. I. Tanner, Numerical study of the Bingham squeeze film problem, J. Non-Newt. Fluid Mech.15 (1984) 75-83. Doi: 10.1016/0377-0257(84)80029-4

[22] T. C. Papanastasiou, Flow of materials with yield, J. Rheol. (31) 1987 385.Doi: $10.1122 / 1.549926$.

[23] N. Kim, Remarks for the axisymmetric Navier-Stokes equations, J. Differential Equations, 187 (2003) 226-239. Doi: 10.1016/S0022-0396(02)00077-3

[24] S. V. Patankar, Numerical Heat Transfer and Fluid Flow, Hemisphere, Washington, D.C., 1980.

[25] N. Quertatani, N. B. Cheikh, B. B. Beya, T. Lili, Numerical simulation of two dimensional Rayleigh- Bénard convection in an enclosure, C. R. Mecanique 336 (2008) 464-470. Doi:10.1016/j.crme.2008.02.004

[26] D. Vola, L. Boscardin, J.C. Latché, Laminar unsteady flows of Bingham fluids: a numerical strategy and some benchmark results, J. Comput. Phys. 187 (2003) 441-456. Doi: 10.1016/S0021-9991(03)00118-9

[27] E. Mitsoulis and T. Zisis, Flow of Bingham plastics in a lid-driven square cavity, J. NonNewt. Fluid Mech. 101 (2001) 173-180. Doi: 10.1016/S0377-0257(01)00147-1 
Table 1: Comparison of the mean Nusselt number $\overline{N u}$ for Newtonian fluid with the benchmark data [25] for square enclosure $(A R=1)$ at $P r=0.71$.

\begin{tabular}{ccc}
\hline$R a$ & Present study & {$[25]$} \\
\hline $1 \times 10^{3}$ & 1.000 & 1.000 \\
$1 \times 10^{4}$ & 2.154 & 2.158 \\
$1 \times 10^{5}$ & 3.907 & 3.910 \\
$1 \times 10^{6}$ & 6.363 & 6.309 \\
\hline
\end{tabular}




\section{FIGURE CAPTIONS}

Figure 1: Schematic diagram of simulation domain: a) CWT, b) CWHF configuration.

Figure 2: Contours of $\theta$ and $\Psi$ with AURs (shown in grey) for $r_{i} / L=1.0, \operatorname{Ra}=10^{5}$ and $\operatorname{Pr}=$ 500 in the CWHF configuration.

Figure 3: Variations of (a) $\theta$ and (b) $W$ with $\left(r-r_{i}\right) / L$ at $z / L=0.5$ at $R a=10^{4}$ and $10^{5}$. (c) Variations of $U$ with $z / L$ at $0.5\left(r_{i}+r_{o}\right) / L$ at $R a=10^{4}$ and $10^{5}$ for $B n=0$ and $B n=0.03$.

Figure 4: Contours of $\theta$ and $\Psi$ with AURs (shown in grey) for $B n=0$ and $B n=0.15$ in case of $r_{i} / L=$ a) 0.25 , b) 1 , c) 4 , d) 16 for $R a=10^{5}$ at $P r=500$ for CWT configuration.

Figure 5: Variation of $\overline{N u}_{c y}$ with $r_{i} / L$ for Newtonian fluids $(B n=0)$. The mean Nusselt number values evaluated from simulation data using Eq. 9 are shown by symbols. The predictions of Eq. 23 are shown by broken lines.

Figure 6: Variation of $\overline{N u}_{c y}$ (evaluated from simulation data using Eq. 9) with $B n$ for Bingham fluids at $R a=10^{5}$ for a) CWT (top row), b) CWHF (bottom row) boundary conditions.

Figure 7: Variation of $\left(B n_{\max }\right)_{c y}$ with $r_{i} / L$ along with the prediction of Eq. 25.

Figure 8: Variation of $\overline{N u}_{c y}$ with $B n$ for different values of $R a$ a) $10^{4}$, b) $5 \times 10^{4}$, c) $10^{5}$ along with the prediction of Eqs. 26ii and 26iii. The mean Nusselt number values evaluated from simulation data using Eq. 9 are shown by symbols. The predictions of Eqs. 26ii and 26iii are shown by continuous lines. 


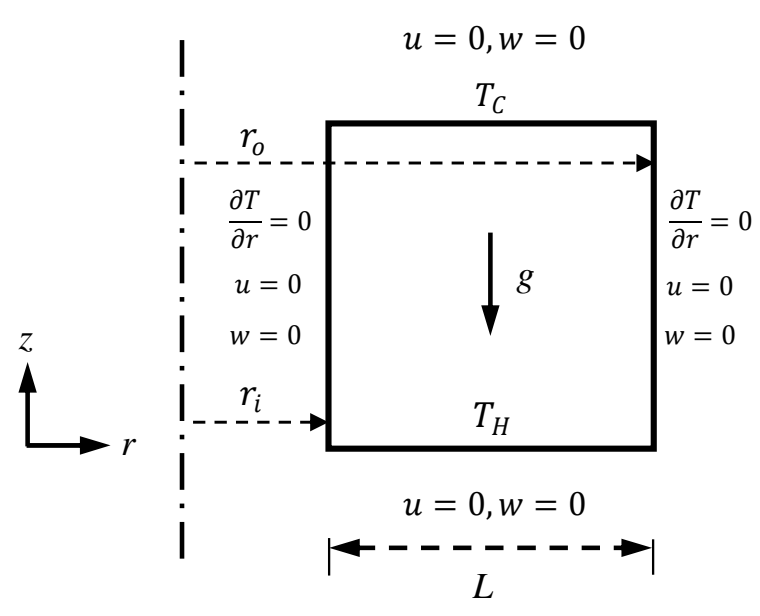

a)

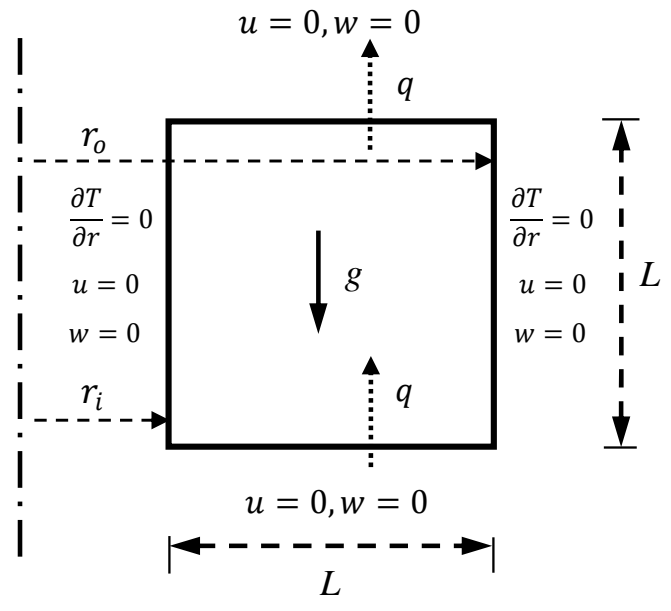

b)

Fig. 1: Schematic diagram of simulation domain: a) CWT, b) CWHF configuration. 


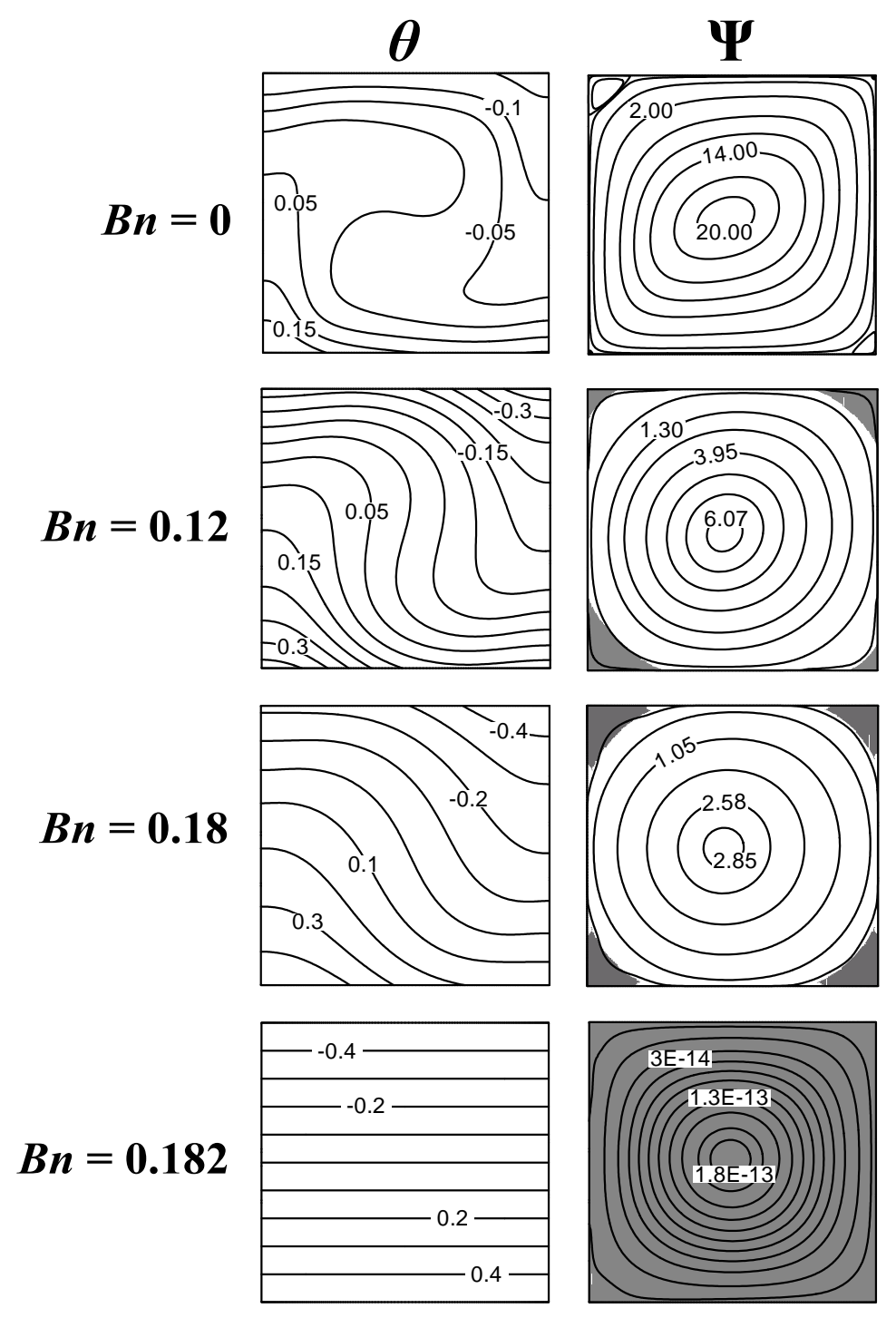

Fig. 2: Contours of $\theta$ and $\Psi$ with AURs (shown in grey) for $r_{i} / L=1.0, R a=10^{5}$ and $P r$ $=500$ in the CWHF configuration. 

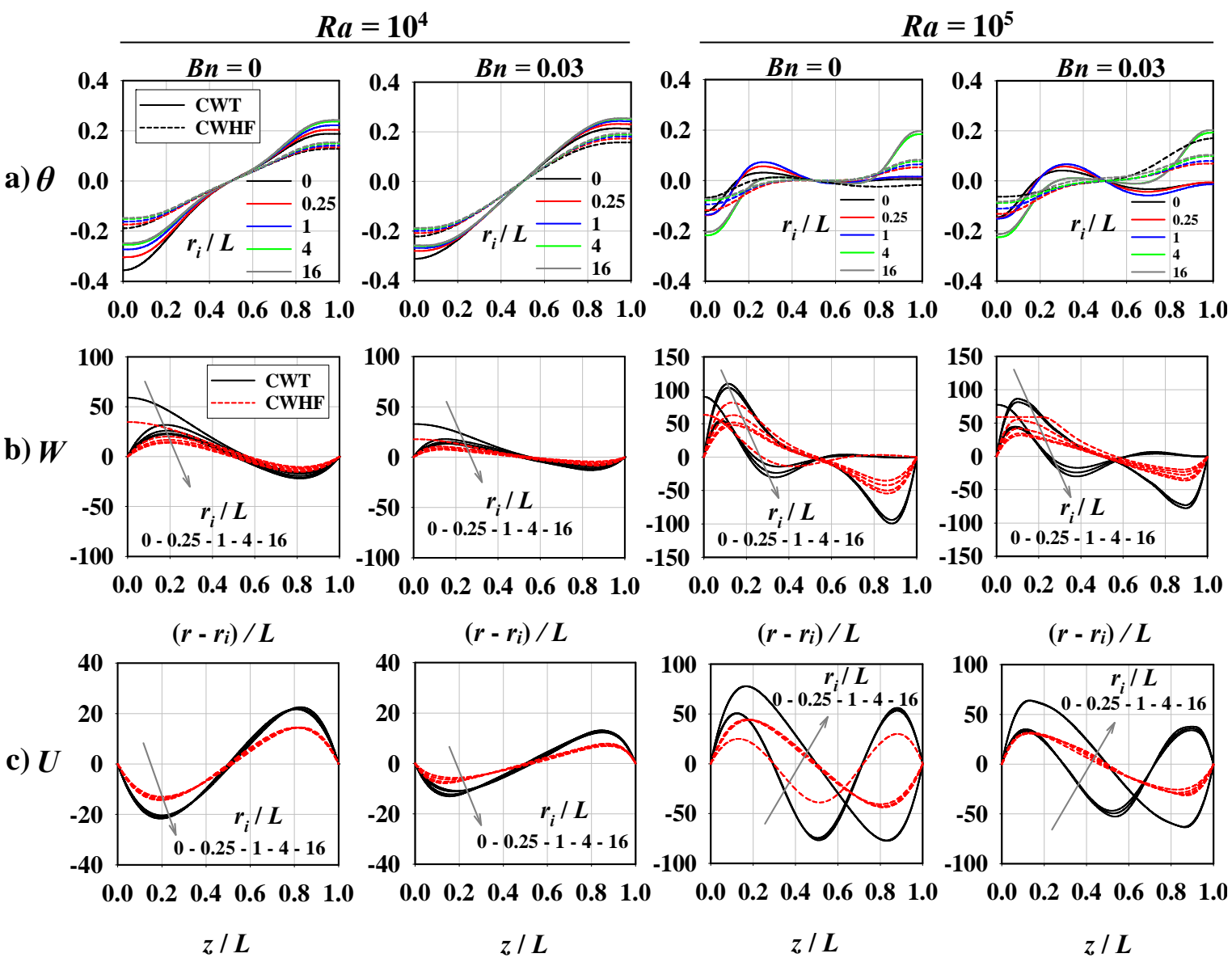

Fig. 3: Variations of (a) $\theta$ and (b) $W$ with $\left(r-r_{i}\right) / L$ at $z / L=0.5$ at $R a=10^{4}$ and $10^{5}$. (c) Variations of $U$ with $z / L$ at $0.5\left(r_{i}+r_{o}\right) / L$ at $R a=10^{4}$ and $10^{5}$ for $B n=0$ and $B n=0.03$. 
a)

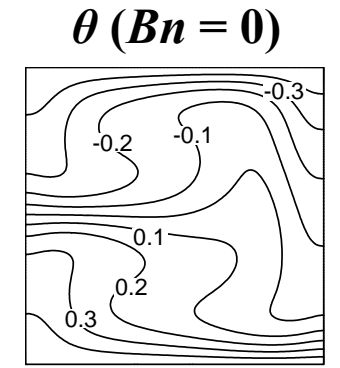

b)

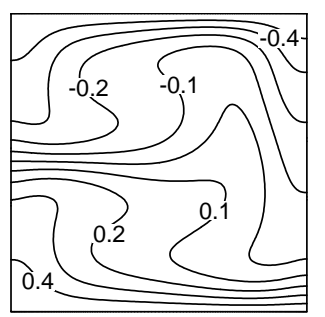

c)

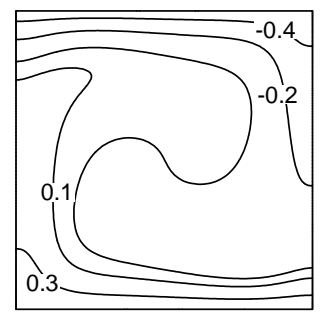

d)

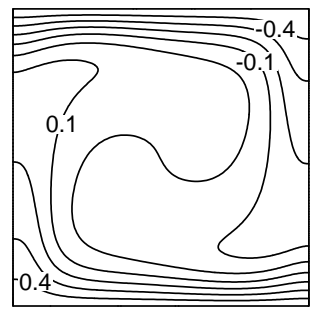

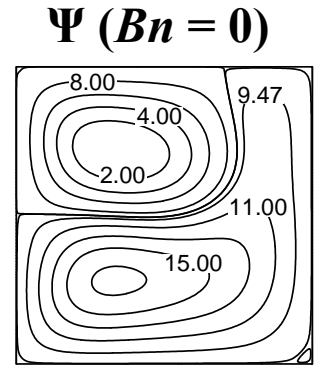
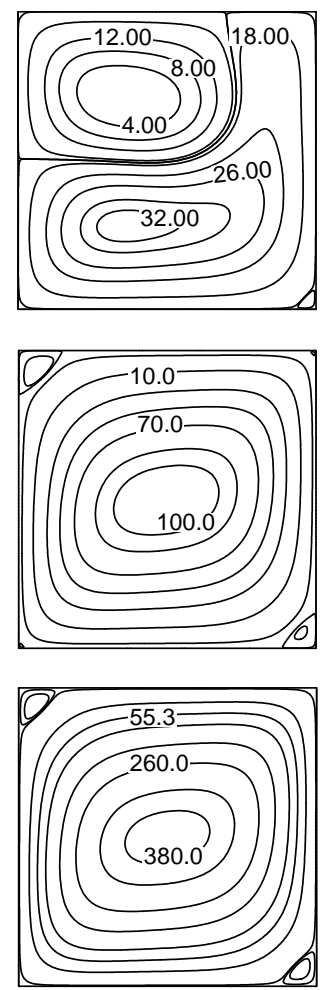
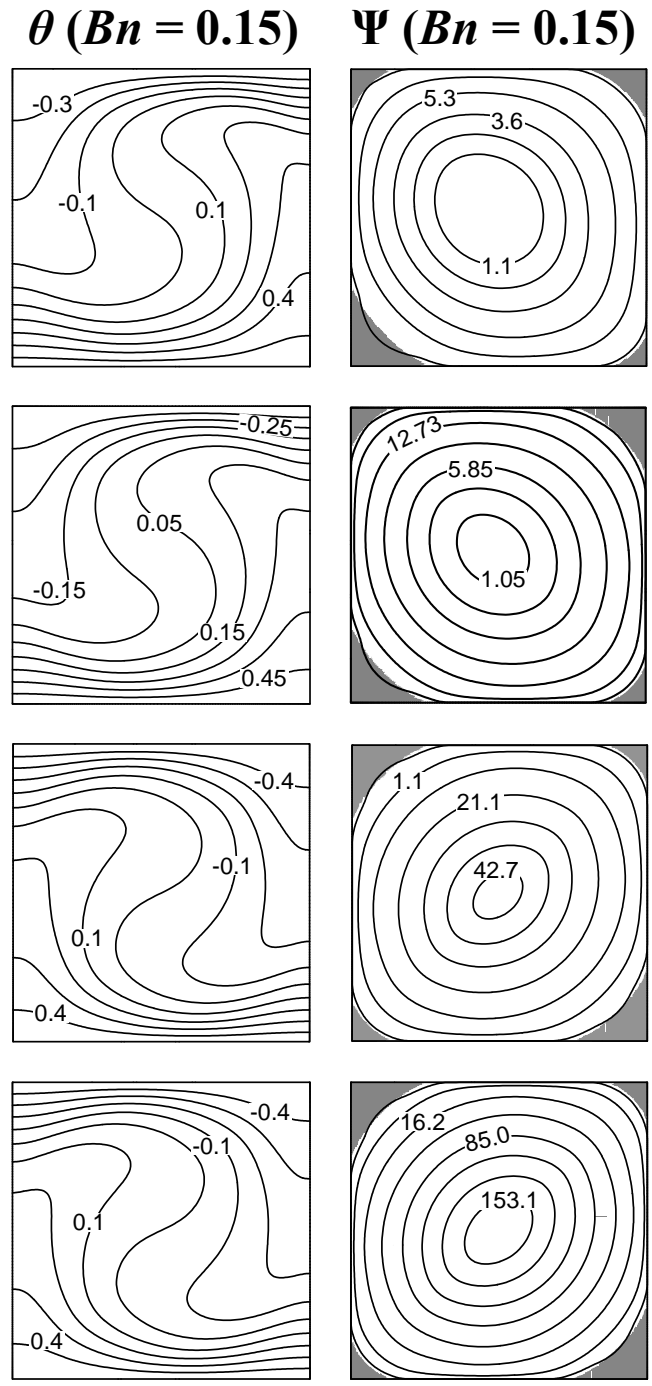

Fig. 4: Contours of $\theta$ and $\Psi$ with AURs (shown in grey) for $B n=0$ and $B n=0.15$ in case of $r_{i} / L=$ a) 0.25 , b) 1 , c) 4 , d) 16 for $R a=10^{5}$ at $P r=500$ for CWT configuration. 

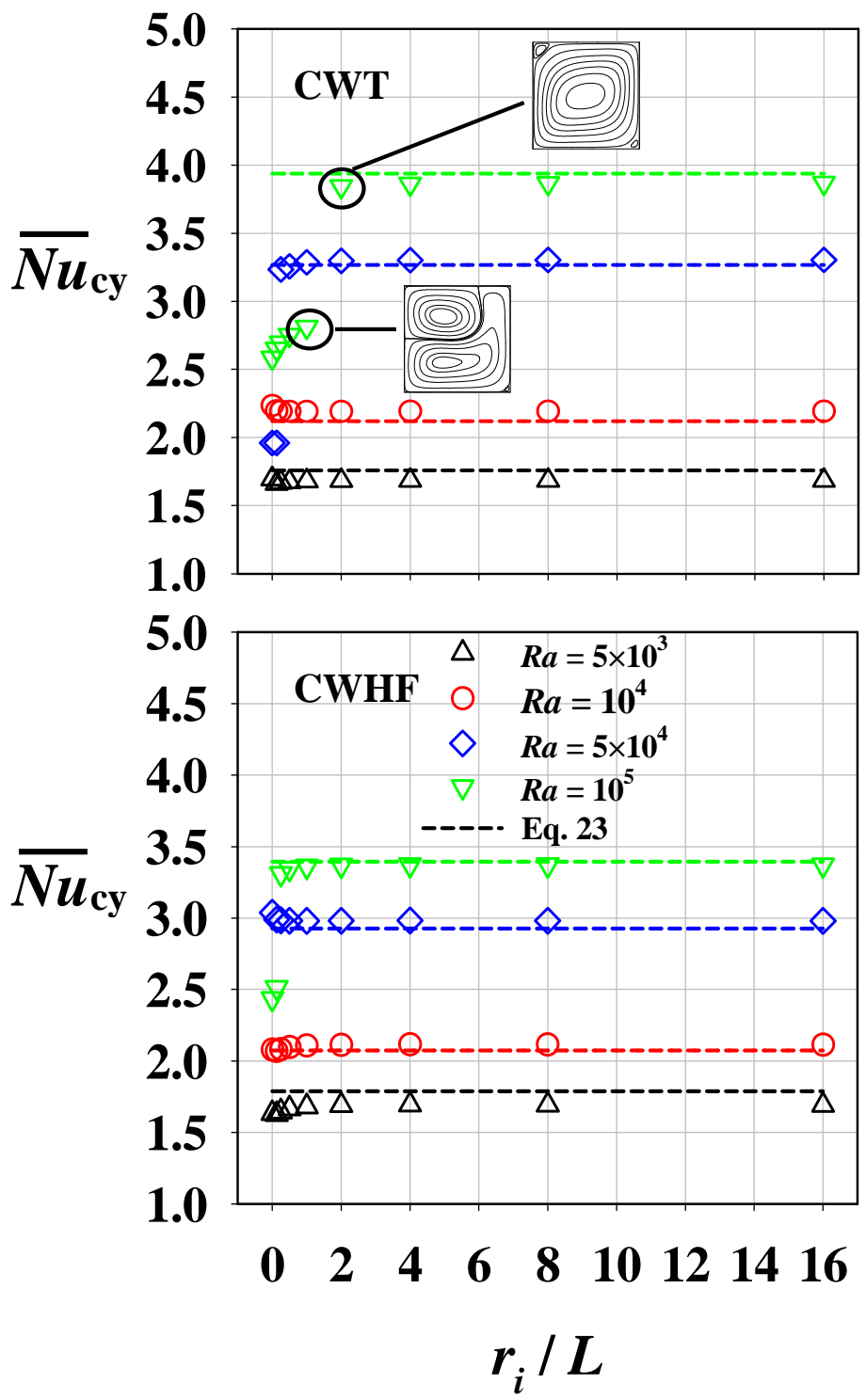

Fig. 5: Variation of $\overline{N u}_{c y}$ with $r_{i} / L$ for Newtonian fluids $(B n=0)$. The mean Nusselt number values evaluated from simulation data using Eq. 9 are shown by symbols. The predictions of Eq. 23 are shown by broken lines. 


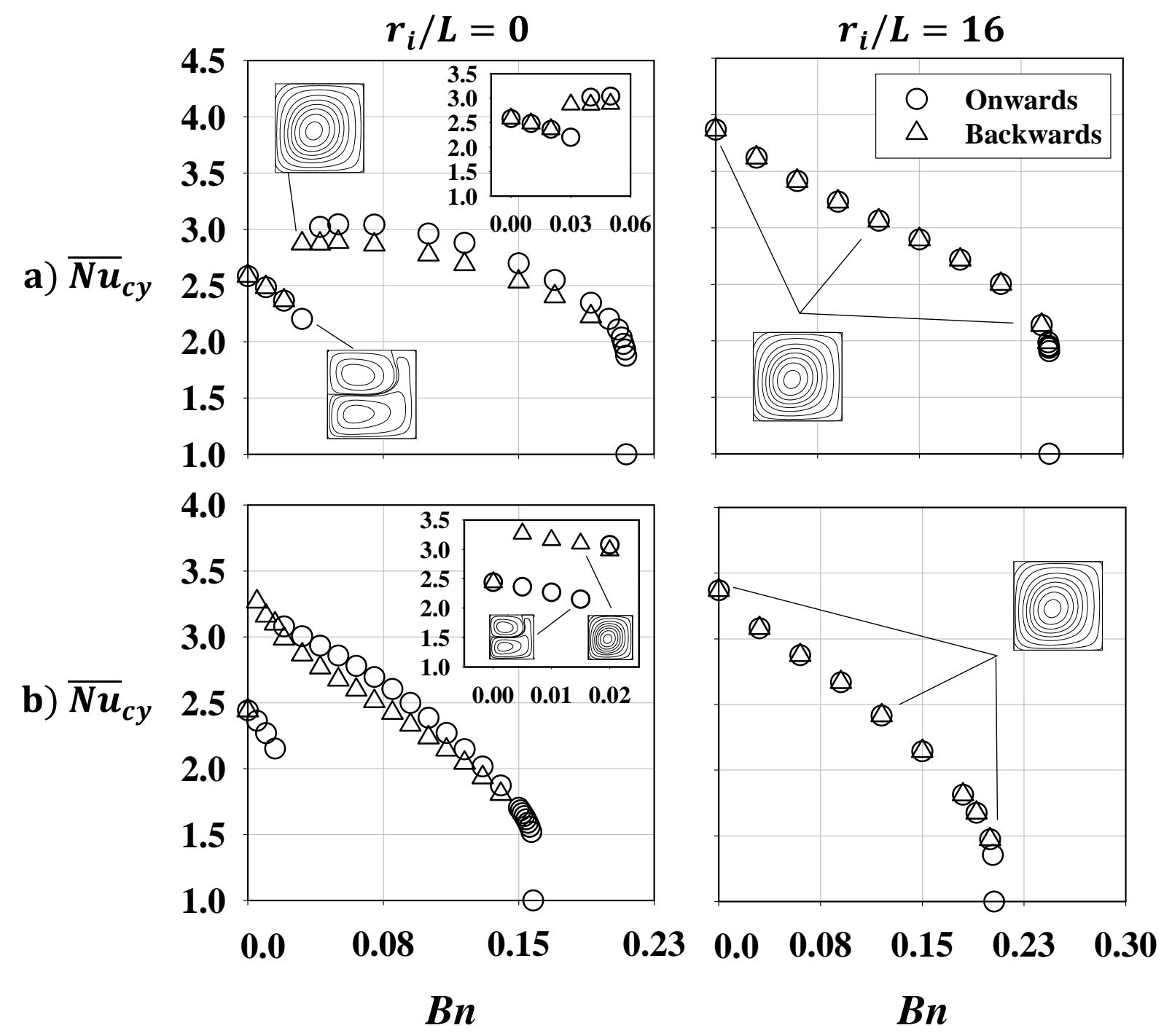

Fig. 6: Variation of $\overline{N u}_{c y}$ (evaluated from simulation data using eq. 9) with Bn for Bingham fluids at $R a=10^{5}$ for a) CWT, b) CWHF boundary conditions. 

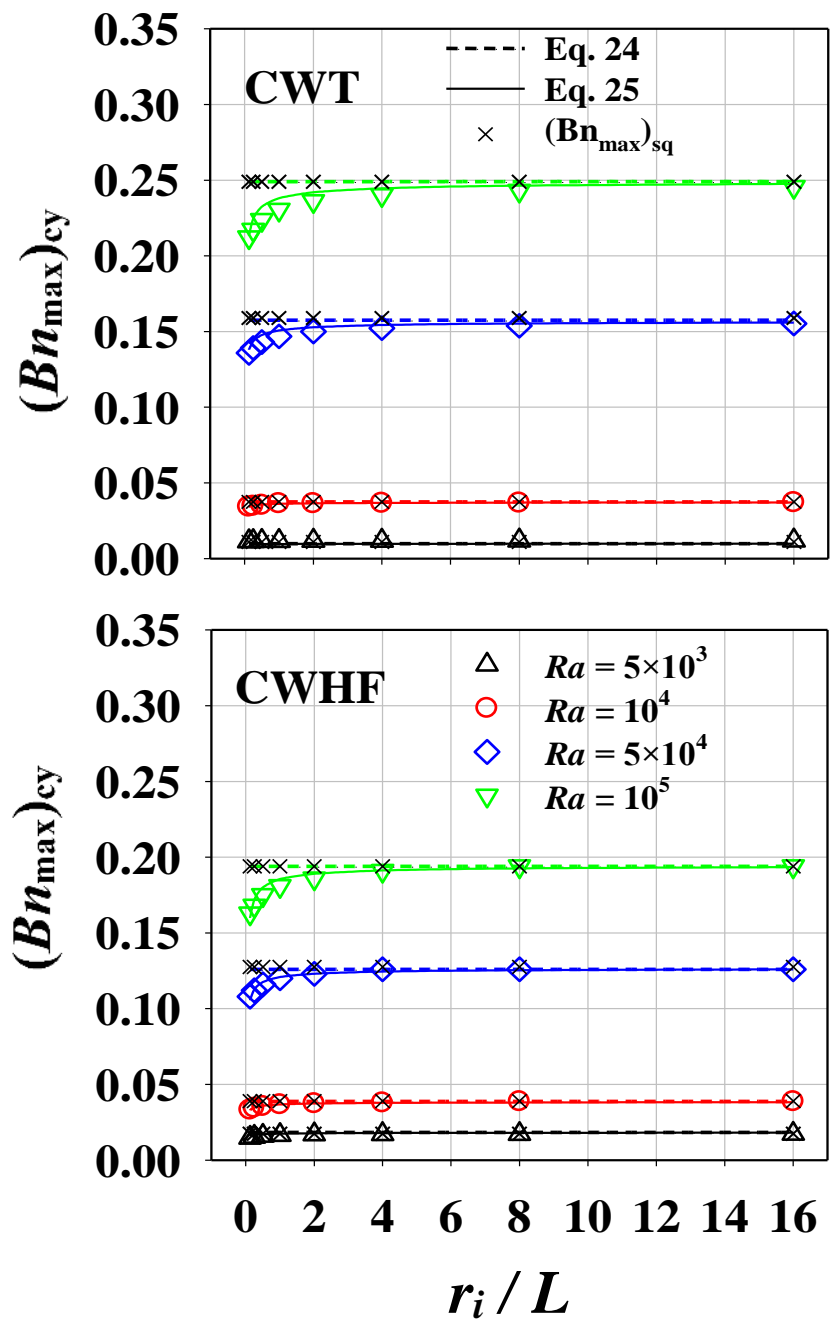

Fig. 7: Variation of $\left(B n_{\max }\right)_{c y}$ with $r_{i} / L$ along with the prediction of Eq. 25. 
a) $\overline{N u}_{\mathrm{cy}}$
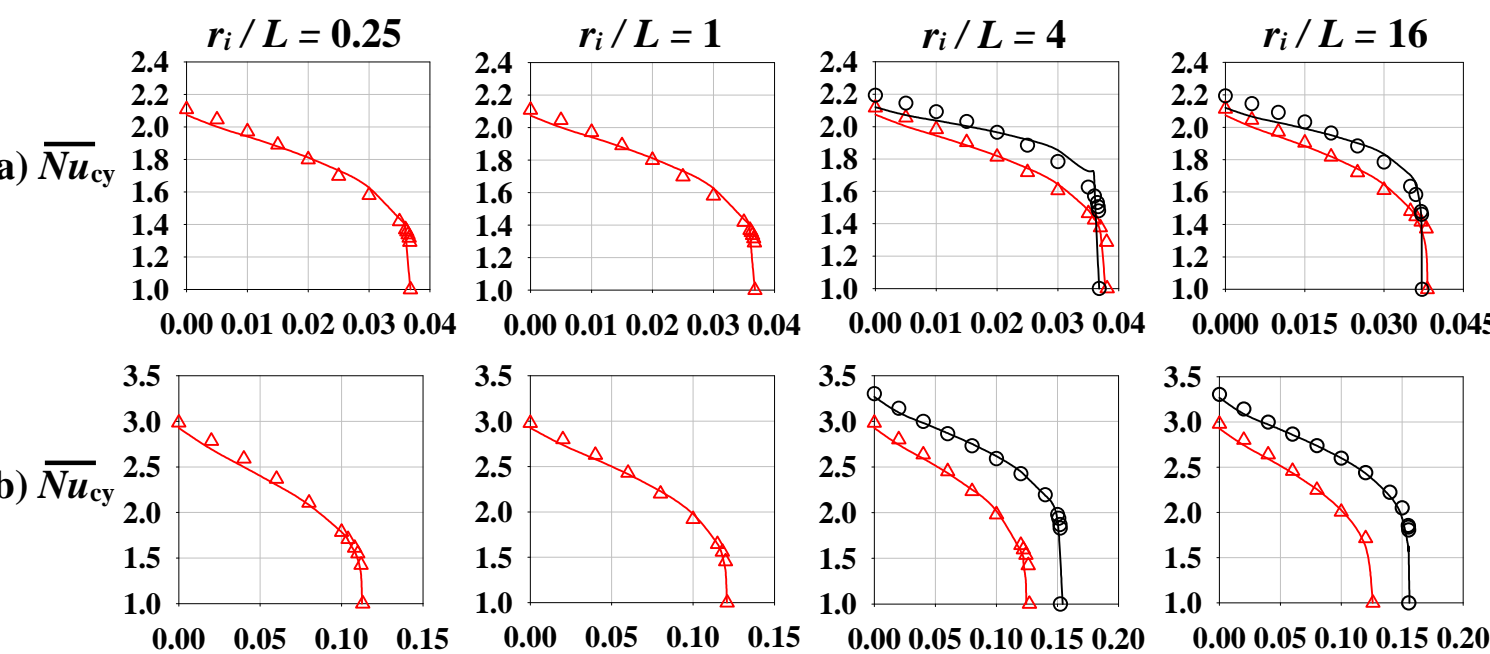

c)

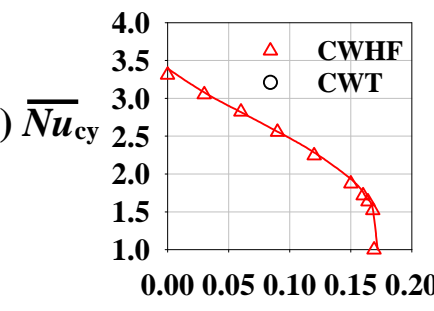

Bn

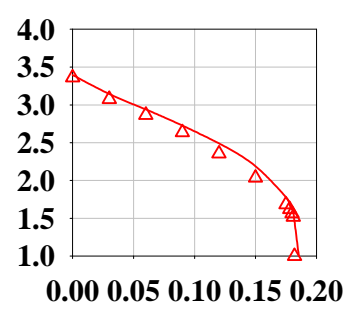

Bn

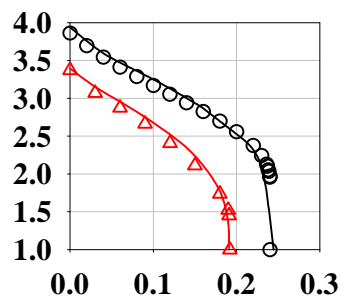

Bn

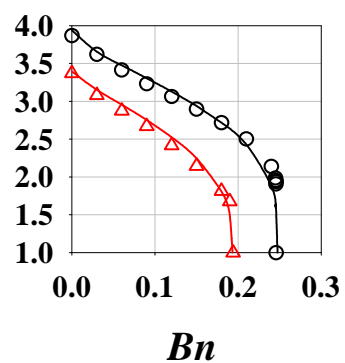

Fig. 8: Variation of $\overline{N u}_{c y}$ with $B n$ for different values of $R a$ : a) $10^{4}$, b)5×104, c) $10^{5}$ along with the prediction of Eqs. 26ii and 26iii. The mean Nusselt number values evaluated from simulation data using Eq. 9 are shown by symbols. The predictions of Eqs. 26ii and 26iii are shown by continuous lines. 